\title{
STATIONARY SOLUTIONS OF CHEMOTAXIS SYSTEMS
}

BY

\author{
RENATE SCHAAF ${ }^{1}$
}

\begin{abstract}
The Keller-Segel Model is a system of partial differential equations modelling a mutual attraction of amoebae caused by releasing a chemical substance (Chemotaxis). This paper analyzes the stationary solutions of the system with general nonlinearities via bifurcation techniques and gives a criterion for bifurcation of stable nonhomogeneous aggregation patterns. Examples are discussed with various kinds of nonlinearities modelling the sensitivity of the chemotaxis response.
\end{abstract}

1. Introduction. In many biological populations cells have the ability to sense spatial inhomogeneities of certain chemicals in their environment and to move towards places of higher concentration (leukocytes, bacteria, gametes, slime mold amoebae). Such oriented migration is called chemotaxis (see [8] for the biological background).

In 1970 E. Keller and L. Segel [10] established a model for the aggregation phase of slime molds. It was known that these amoebae are attracted by a chemical, cyclic AMP, which is produced by themselves and can be degraded by some enzyme present in the cells. The Keller-Segel Model, described below, is based on these mechanisms: chemotactic response of cells to a chemical whose production and degradation depends on the concentration of the cells and the chemical. The aim of this paper is to show some mathematical implications of basic model assumptions like this.

One may consider the cell flux to consist of a random migration (diffusion) part and a drift in the direction of the chemical gradient. Assuming the whole process to take place on a suitable bounded region $\Omega \subset \mathbf{R}^{n}$ with no flux across the boundary, and that birth and death of cells can be ignored, one has the following equation for the cell density $u=u(x, t),(x, t) \in \Omega \times \mathbf{R}^{+}$, and the density $v=v(x, t)$ of the chemoattractant:

$$
\begin{aligned}
& \partial_{t} u=\nabla \cdot(\mu(u, v) \nabla u-\chi(u, v) \nabla v) \quad \text { on } \Omega \times \mathbf{R}^{+}, \\
& \mu(u, v) \partial_{\nu} u-\chi(u, v) \partial_{\nu} v=0 \quad \text { on } \partial \Omega \times \mathbf{R}^{+} .
\end{aligned}
$$

$\left(\nu\right.$ is the outward normal on $\partial \Omega, \nabla=\left(\partial / \partial x_{1}, \ldots, \partial / \partial x_{n}\right)$.)

The random motility coefficient $\mu>0$ may depend on $u$ and $v$ allowing e.g. the possibility that the general motility is enhanced in presence of the chemical (chemokinesis), or is diminished at high cell concentrations.

Received by the editors December 18, 1984.

1980 Mathematics Subject Classification. Primary 35B32, 35B35; Secondary 34B12, 92A18.

Key words and phrases. Chemotaxis, Keller-Segel Model, cross diffusion, stationary solutions, bifurcation, global bifurcation, time map, exchange of stability

${ }^{1}$ This work was supported by the Deutsche Forschungsgemeinschaft. 
Some plausible form of the chemotaxis coefficient $\chi>0$ can be obtained by the following reasoning: By some receptor mechanism, cells do not measure the gradient of $v$ but of some $\phi(v)$ with a sensitivity function $\phi, \phi^{\prime}>0$ (see [12]). The velocity of a single cell moving up the gradient of $\phi(v)$ may be proportional to the random motility coefficient $\mu(u, v)$, such that the total chemotaxic flux is $\chi_{0} \mu(u, v) u \nabla \phi(v)$, i.e.

$$
\chi(u, v)=\chi_{0} \mu(u, v) u \phi^{\prime}(v) .
$$

For a detailed derivation of (1.1) and (1.2) as a diffusion approximation to a biased random walk model, see [2 and $\mathbf{3}$ ].

Several normalized forms of $\phi$ have been suggested

$$
\begin{gathered}
\phi(v)=v \quad(\text { direct measurement }), \\
\phi(v)=\ln (c+v) \quad(\text { logarithmic sensitivity, } c \geqslant 0), \\
\phi(v)=v /(1+c v) \quad(\text { Michaelis-Menten receptor kinetics), } \\
\phi(v)=v^{2} /\left(1+c v^{2}\right) \quad(\text { cooperative binding, } c>0) .
\end{gathered}
$$

If the production and degradation of the chemical $v$ depends only on $u$ and $v$ (e.g. if the cells produce the chemical in order to attract others) one gets

$$
\partial_{t} v=\nu_{0} \Delta v+k(u, v) \quad \text { on } \Omega \times \mathbf{R}^{+},
$$

$\nu_{0}>0$ being a constant diffusion coefficient, $k: \mathbf{R}^{+} \times \mathbf{R}^{+} \rightarrow \mathbf{R}$ modelling the reactions leading to production and degradation of $v$. No flux condition on $\partial \Omega$ gives

$$
\partial_{\nu} v=0 \text { on } \partial \Omega \text {. }
$$

(1.1), (1.7), (1.8) conbined give the following chemotaxis system with homogeneous Neumann conditions:

$$
\begin{aligned}
& \partial_{t} u=\nabla \cdot(\mu(u, v) \nabla u-\chi(u, v) \nabla v), \\
& \partial_{t} v=\nu_{0} \Delta v+k(u, v), \\
& \left.\partial_{\nu} u\right|_{\partial \Omega}=\left.\partial_{\nu} v\right|_{\partial \Omega}=0 .
\end{aligned}
$$

Equations (1.9) have been stated in 1970 by E. F. Keller and L. A. Segel [10] to model the aggregation phase of slime mold amoebae.

Whenever production can equate degradation $\left(k^{-1}(\{0\}) \neq \varnothing\right)$ there is a set of spatially homogeneous solutions to (1.9) which (under reasonable conditions) are stable for low cell densities. Segel and Keller argue that aggregation is initiated when the total cell mass exceeds a certain critical value for which the homogeneous distribution is no longer stable.

One step towards the examination of the further time development is to look for bifurcating steady states which have a spatial structure and eventually become stable.

In this paper we will apply local and global bifurcation techniques to the stationary system of (1.9) which turns out to be equivalent to a parameter dependent scalar equation

$$
\Delta v+f(v, \lambda)=0, \quad \partial_{v} v \mid \partial \Omega=0,
$$

with $f(v, \lambda)=\left(1 / \nu_{0}\right) k(\varphi(v, \lambda), v)$. The parameter dependent function $\varphi(v, \lambda)$ is obtained from $\mu$ and $\chi$ by solving a first order ODE (see $\$ 2$ ). 
In [16] we have examined global and local bifurcation of solutions to (1.10) in one space dimension $n=1$, where some of the results can obviously be carried over to dimensions $n>1$.

With this we can characterize bifurcation of spatially inhomogeneous solutions to (1.8) rather simply by conditions along the trivial set $M:=\{(v, \lambda) \mid v \equiv m, f(m, \lambda)$ $=0\}$. Results can be found in $\$ 3$.

$\S 4$ contains the discussion of stability. For this question one has to consider the full system (1.9) again. But it turns out that criteria for stability of homogeneous distributions and exchange of stability at a bifurcation point can be formulated in terms of $f$ and $\varphi$ in the case of one space dimension.

In the last section we will discuss examples for $\chi$ as in (1.2) with sensitivity functions $\phi$ as in (1.3)-(1.5) and $k$ being as simple as possible. In all of these examples we can apply results for the so-called time map, proved in [16 and 17], in order to get the whole global bifurcation behaviour of the systems. In each case the bifurcating branches can be shown to be smooth curves which in some examples form closed loops joining two bifurcation points, an example for the second alternative of Rabinowitz's global theorem [15] being verified.

In all cases the result of $\S 4$ concerning exchange of stability can be applied, at least within a certain parameter range.

As a first step towards an investigation of stability along the whole branch we can show that a region of stability can only end at a turning point or a Hopf bifurcation point.

2. Reduction to a single equation. For the data of the stationary system

$$
\begin{aligned}
& \nabla \cdot(\mu(u, v) \nabla u-\chi(u, v) \nabla v)=0 \\
& \nu_{0} \Delta v+k(u, v)=0 \\
& \left.\partial_{\nu} u\right|_{\partial \Omega}=\left.\partial_{\nu} v\right|_{\partial \Omega}=0
\end{aligned}
$$

we shall henceforth assume the following conditions:

$$
\Omega \subset \mathbf{R}^{n} \text { is a bounded open region with smooth boundary, }
$$
and the ODE

$$
\mu, \chi: \mathbf{R}^{+} \times \mathbf{R}^{+} \rightarrow \mathbf{R}^{+} \text {are twice continuously differentiable }
$$

As Banach spaces for the problem we introduce

$$
\begin{aligned}
& \dot{r}(s)=\chi(r, s) / \mu(r, s) \text { has a unique solution } r: \mathbf{R}^{+} \rightarrow \mathbf{R}^{+} \\
& \text {for any initial condition } r\left(s_{0}\right)=r_{0}, s_{0}, r_{0} \in \mathbf{R}^{+}, \\
& \quad k: \mathbf{R}^{+} \times \mathbf{R}^{+} \rightarrow \mathbf{R} \text { is twice continuously differentiable } \\
& \quad \text { and } k^{-1}(\{0\}) \neq \varnothing .
\end{aligned}
$$

$$
\begin{aligned}
& Y= \begin{cases}C^{0, \alpha}(\bar{\Omega}, \mathbf{R}) & \text { for } n>1, \\
C^{0}(\bar{\Omega}, \mathbf{R}) & \text { for } n=1,\end{cases} \\
& Z= \begin{cases}C^{2, \alpha}(\bar{\Omega}, \mathbf{R}) & \text { for } n>1, \\
C^{2}(\bar{\Omega}, \mathbf{R}) & \text { for } n=1(0<\alpha<1),\end{cases}
\end{aligned}
$$




$$
X:=\left\{u \in Z\left|\partial_{\nu} u\right|_{\partial \Omega}=0\right\}
$$

with their usual norms.

As we consider positive solutions only, we set

$$
B:=\left\{u \in X \mid u(\bar{\Omega}) \subset \mathbf{R}^{+}\right\} .
$$

$B$ is an open subset of $X$.

Let $\varphi(x, \lambda)$ be the "flux" of (2.4), i.e.

$$
\varphi(s, \lambda)=r(s) \quad \text { with } \quad \dot{r}(s)=(\chi / \mu)(r, s), r(1)=\lambda .
$$

Let $\partial_{1}\left(\partial_{2}\right)$ denote the derivatives with respect to the first (second) argument. Then $\varphi$ satisfies

$$
\begin{gathered}
\partial_{1} \varphi(s, \lambda)=(\chi / \mu)(\varphi(s, \lambda), s), \\
\partial_{1} \partial_{2} \varphi(s, \lambda)=\left(\partial_{1} \chi / \mu\right)(\varphi(s, \lambda), s) \partial_{2} \varphi(s, \lambda) .
\end{gathered}
$$

So

$$
\partial_{2} \varphi(s, \lambda)>0 \text { for all } s, \lambda \in \mathbf{R}^{+},
$$

since $\partial_{2} \varphi(\cdot, \lambda)$ solves a linear ODE with $\partial_{2} \varphi(1, \lambda)=1>0$. With this we can reduce (2.1) to a scalar problem. (For a similar reduction see $[21]$ or $[5,13]$ for a special case.)

2.1 Theorem. $(u, v) \in B \times B$ is a solution of (2.1) iff, for some $\lambda \in \mathbf{R}^{+}$,

$$
u(x)=\varphi(v(x), \lambda) \quad \text { for all } x \in \bar{\Omega}
$$

and

$$
\nu_{0} \Delta v+k(\varphi(v, \lambda), v)=0 .
$$

Proof. Let $(u, v) \in B \times B$ be a solution of (2.1). Then there is a positive function $\psi \in C^{2}(\bar{\Omega})$ with

$$
u(x)=\varphi(v(x), \psi(x)) \text { for all } x \in \bar{\Omega} .
$$

This is because, for any $x \in \bar{\Omega}$, (2.4) has a unique solution $r_{x}: \mathbf{R}^{+} \rightarrow \mathbf{R}^{+}$with $r_{x}(v(x))=u(x)$. Setting $\psi(x)=r_{x}(1)$ gives the desired $C^{2}$-function $\psi \cdot \psi$ satisfies the Neumann boundary conditions since, on $\partial \Omega$,

$$
0=\partial_{\nu} u=\partial_{1} \varphi(v, \psi) \partial_{\nu} v+\partial_{2} \varphi(v, \psi) \partial_{\nu} \psi=\partial_{2} \varphi(v, \psi) \partial_{\nu} \psi
$$

with $\partial_{2} \varphi(v, \psi) \neq 0$ because of (2.12).

From the first equation of (2.1) we get, with (2.10), (2.15),

$$
\begin{aligned}
0 & =\nabla \cdot(\mu(u, v) \nabla u-\chi(u, v) \nabla v) \\
& =\nabla \cdot\left(\mu(u, v)\left(\partial_{1} \varphi(v, \psi) \nabla v+\partial_{2} \varphi(v, \psi) \nabla \psi\right)-\chi(u, v) \nabla v\right) \\
& =\nabla \cdot\left(\mu(u, v) \partial_{2} \varphi(v, \psi) \nabla \psi\right) .
\end{aligned}
$$

Thus $\psi$ solves an elliptic PDE

$$
\Delta \psi+\sum_{i=1}^{u} c_{i} \partial_{i} \psi=0,\left.\quad \partial_{\nu} \psi\right|_{\partial \Omega}=0,
$$

with coefficients $c_{i}$ being continuous on $\bar{\Omega}$. Then $\psi \equiv$ const $=: \lambda$ by the Hopf maximum principle [14], and (2.13), (2.14) follow. 
The "if" part of the theorem follows by direct calculation. We notice that for the special form of $\chi$ as in (1.2) we get

$$
\varphi(s, \lambda)=\lambda c e^{\chi_{0} \phi(s)}
$$

with $c=e^{-\chi_{0} \phi(1)}$. From now on we will define

$$
f(s, \lambda):=\left(1 / \nu_{0}\right) k(\varphi(s, \lambda), s) \text { for } s, \lambda>0 .
$$

Then Theorem 2.2 says that solving (1.2) in $B \times B$ is equivalent to solving

$$
\Delta v+f(v, \lambda)=0
$$

for $(v, \lambda) \in B \times \mathbf{R}^{+}$.

Next we will note how the linearized equation (2.1) around a solution $\left(u_{0}, v_{0}\right)=$ $\left(\varphi\left(v_{0}, \lambda_{0}\right), v_{0}\right) \in B \times B$ can also be reduced to a scalar problem.

2.2 THEOREM. In addition to (2.3) let $\mu, \chi$ be three times continuously differentiable. Let $\left(u_{0}, v_{0}\right) \in B \times B$ be a solution of $(2.1)$, that is, $\left(u_{0}, v_{0}\right)=\left(\varphi\left(v_{0}, \lambda_{0}\right), v_{0}\right)$, by Theorem 2.1 for some $\lambda_{0}>0$. Let $G: B \times B \rightarrow Y \times Y$ be defined by the left-hand side of equation (2.1). Then $(u, v) \in X \times X$ is a solution of the linearized equation

$$
D G\left(u_{0}, v_{0}\right)(u, v)=0
$$

iff

$$
u(x)=\partial_{1} \varphi\left(v_{0}(x), \lambda_{0}\right) v(x)+\lambda \partial_{2} \varphi\left(v_{0}(x), \lambda_{0}\right)
$$

for some $\lambda \in \mathbf{R}$ and

$$
\Delta v+\partial_{1} f\left(v_{0}, \lambda_{0}\right) v+\lambda \partial_{2} f\left(v_{0}, \lambda_{0}\right)=0
$$

with $f$ defined by (2.17).

Proof. Suppose $D G\left(u_{0}, v_{0}\right)(u, v)=0$. Then there is a function $\psi \in C^{2}(\bar{\Omega})$ with

$$
u(x)=\partial_{1} \varphi\left(v_{0}(x), \lambda_{0}\right) v(x)+\psi(x) \partial_{2} \varphi\left(v_{0}(x), \lambda_{0}\right)
$$

since $\partial_{1} \varphi, \partial_{2} \varphi$ are in $C^{2}$ with $\partial_{2} \varphi>0$. Then

$$
\begin{aligned}
0= & D G\left(u_{0}, v_{0}\right)(u, v) \\
= & \nabla \cdot\left(\partial_{1} \mu\left(u_{0}, v_{0}\right) \nabla u_{0} u+\partial_{2} \mu\left(u_{0}, v_{0}\right) \nabla v_{0} v+\mu\left(u_{0}, v_{0}\right) \nabla u\right. \\
& \left.\quad-\partial_{1} \chi\left(u_{0}, v_{0}\right) \nabla v_{0} u-\partial_{2} \chi\left(u_{0}, v_{0}\right) \nabla v_{0} v-\chi\left(u_{0}, v_{0}\right) \nabla v\right) .
\end{aligned}
$$

By Theorem 2.1 we have $u_{0}=\varphi\left(v_{0}, \lambda_{0}\right)$ with

$$
\mu(\varphi(s, \lambda), s) \partial_{1} \varphi(s, \lambda)=\chi(\varphi(s, \lambda), s)
$$

from (2.10). Differentiating (2.24) with respect to $s$ and $\lambda$ gives

$$
\begin{aligned}
\partial_{1} \mu(\varphi(s, \lambda), s)\left(\partial_{1} \varphi\right. & (s, \lambda))^{2} \\
+ & \partial_{2} \mu(\varphi(s, \lambda), s) \partial_{1} \varphi(s, \lambda)+\mu(\varphi(s, \lambda), s) \partial_{1}^{2} \varphi(s, \lambda) \\
& =\partial_{1} \chi(\varphi(s, \lambda), s) \partial_{1} \varphi(s, \lambda)+\partial_{2} \chi(\varphi(s, \lambda), s)
\end{aligned}
$$

and

$$
\begin{gathered}
\partial_{1} \mu(\varphi(s, \lambda), s) \partial_{2} \varphi(s, \lambda) \partial_{1} \varphi(s, \lambda)+\mu(\varphi(s, \lambda), s) \partial_{1} \partial_{2} \varphi(s, \lambda) \\
=\partial_{1} \chi(\varphi(s, \lambda), s) \partial_{2} \varphi(s, \lambda) .
\end{gathered}
$$


Using this and expression (2.22) for $u$ we get from (2.23)

$$
0=\nabla \cdot\left(\mu\left(u_{0}, v_{0}\right) \partial_{2} \varphi\left(u_{0}, v_{0}\right) \nabla \psi\right) .
$$

Thus again as in Theorem $2.1 \psi \equiv$ const $:=\lambda$ by the Hopf maximum principle which gives (2.20). (2.21) then results from the second equation for $(u, v)$. The converse of the theorem's assertion follows by differentiating $0=G_{1}(\varphi(v, \lambda), v)$ for all $(v, \lambda) \in B \times \mathbf{R}^{+}$.

3. Bifurcation of nonhomogeneous solutions. In this paragraph we will briefly collect bifurcation results for the parameter-dependent problem

$$
\Delta v+f(v, \lambda)=0,\left.\quad \partial_{v} v\right|_{\partial \Omega}=0,
$$

with

$$
f(s, \lambda)=\left(1 / \nu_{0}\right) k(\varphi(s, \lambda), s),
$$

which has been proved to be equivalent to the stationary system (2.1) in the last section (see (2.9), (2.13), (2.14)). Results are applications of existing local and modified global theorems on bifurcation from simple eigenvalues $[6,1]$. Proofs can be found in [16].

We define the trivial set of constant solutions

$$
M:=\left\{(m, \lambda) \in \mathbf{R}^{+} \times \mathbf{R}^{+} \mid f(m, \lambda)=0\right\}
$$

with

$$
M_{c}:=\{(m, \lambda) \in M \mid \nabla f(m, \lambda)=0\} .
$$

We consider $M$ to be a subset of $B \times \mathbf{R}^{+}$by identifying constant functions with their values. The nontrivial solution set shall be denoted by

$$
S:=\left\{(v, \lambda) \in B \times \mathbf{R}^{+} \mid(v, \lambda) \text { solves (3.1), } v \text { nonconstant }\right\} .
$$

$0=\mu_{0}<\mu_{1} \leqslant \mu_{2} \leqslant \cdots$ are the eigenvalues of the operator $v \rightarrow \Delta v$ between $X$ and $Y$ corresponding to a complete orthogonal (in $L^{2}$ ) sequence of eigenfunctions $\left(\omega_{j}\right)_{j \in \mathbf{N}}$.

For one space dimension, $n=1$, we will show that all solutions in a bifurcating global branch stay in the same "pattern class", and therefore introduce, for $j \in \mathbf{N}$, $n=1, X=\left\{v \in C^{2}(\bar{\Omega})\left|v^{\prime}\right|_{\partial \Omega}=0\right\}$,

(3.3) $O_{j}:=\left\{v \in X \mid v^{\prime}\right.$ has exactly $j+1$ zeros in $\bar{\Omega}$ all of which are simple $\}$.

(A zero $x_{0}$ of a $C^{1}$-function $g: \Omega \subset \mathbf{R} \rightarrow \mathbf{R}$ is called simple if $g^{\prime}\left(x_{0}\right) \neq 0$.) The $O_{j}$ are open subsets of $X$.

3.1 THEOREM. (a) If $\left(m_{0}, \lambda_{0}\right) \in M$ is a bifurcation point of (3.1) then necessarily

$$
\left(m_{0}, \lambda_{0}\right) \in M_{c} \text { or } \partial_{1} f\left(m_{0}, \lambda_{0}\right)=\mu_{j} \text { for some } j \geqslant 1 .
$$

(Note that $\mu_{j}>0$; so near a possible bifurcation point in $M \backslash M_{c}$ the set $M$ is always parametrizable over $\lambda$.)

(b) If for some $\left(m_{0}, \lambda_{0}\right) \in M$

$$
\partial_{1} f\left(m_{0}, \lambda_{0}\right)=\mu_{j} \text { with } j \geqslant 1, \mu_{j} \text { being a simple eigenvalue, }
$$

then let $\lambda \rightarrow(m(\lambda), \lambda)$ be a parametrization of $M$ near $\left(m_{0}, \lambda_{0}\right)$. If, in addition,

$$
\left.\frac{d}{d \lambda}\left(\partial_{1} f(m(\lambda), \lambda)\right)\right|_{\lambda=\lambda_{0}} \neq 0,
$$


then the following assertions hold:

(i) $\left(m_{0}, \lambda_{0}\right)$ is a bifurcation point, i.e. $\left(m_{0}, \lambda_{0}\right) \in \bar{S}$.

(ii) Let $C\left(m_{0}, \lambda_{0}\right)$ be the connected component of $\bar{S}$ with $\left(m_{0}, \lambda_{0}\right)$. Then $C\left(m_{0}, \lambda_{0}\right)$ is not relatively compact in $B \times \mathbf{R}^{+}$or $C\left(m_{0}, \lambda_{0}\right)$ contains a point $\left(m_{1}, \lambda_{1}\right) \in M$ different from $\left(m_{0}, \lambda_{0}\right)$.

(iii) In a neighborhood $U=U\left(m_{0}, \lambda_{0}\right) \subset B \times \mathbf{R}^{+}$the nontrivial branch $C\left(m_{0}, \lambda_{0}\right)$ is a $C^{1}$-curve

$$
\varepsilon \in]-\varepsilon_{0}, \varepsilon_{0}\left[\mapsto(V(\varepsilon), \Lambda(\varepsilon)) \text { with } V^{\prime}(0)=\omega_{j}\right.
$$

For space dimension $n=1$ additionally holds.

(iv) If $M_{c}=\varnothing$ then nodal properties of $v^{\prime}$ are conserved along the whole branch, i.e. $C\left(m_{0}, \lambda_{0}\right) \backslash M \subset O_{j} \times \mathbf{R}^{+}$and $\partial_{1} f(\tilde{m}, \tilde{\lambda})=\mu_{j}$ for all bifurcation points $(\tilde{m}, \tilde{\lambda}) \in$ $C\left(m_{0}, \lambda_{0}\right) \cap M$.

Note that for $n=1$ all eigenvalues $\mu_{j}$ of $v \mapsto v^{\prime \prime}$ are simple. As an illustration of Theorem 3.1 let us consider a simple example.

3.2 EXAmple. Assume (1.2) for $\chi$ with $\phi(v)=v, k(u, v)=\nu_{0}(\alpha u-\beta v)$. That is, the first equation of (2.1) is solved by $u=\lambda e^{\chi_{0} v}$ (see (2.16); the factor $c$ can be omitted without loss of generality), and we have to consider

$$
\Delta v+f(v, \lambda)=0, \quad(v, \lambda) \in B \times \mathbf{R}^{+}
$$

with $f(s, \lambda)=\alpha \lambda e^{\chi_{0} s}-\beta s$.

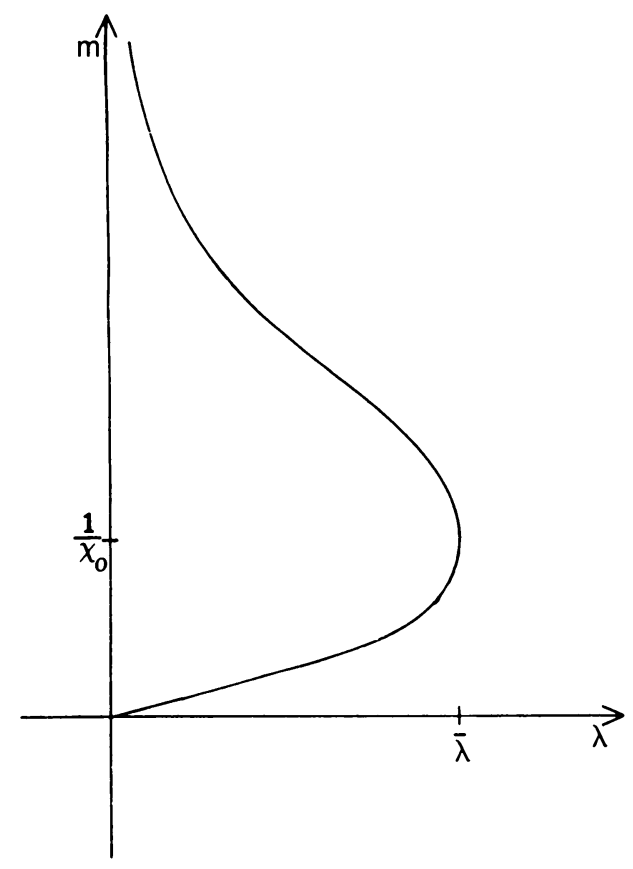

$$
M=\{(m, \lambda) \mid f(m, \lambda)=0\}
$$

Figure 3.1

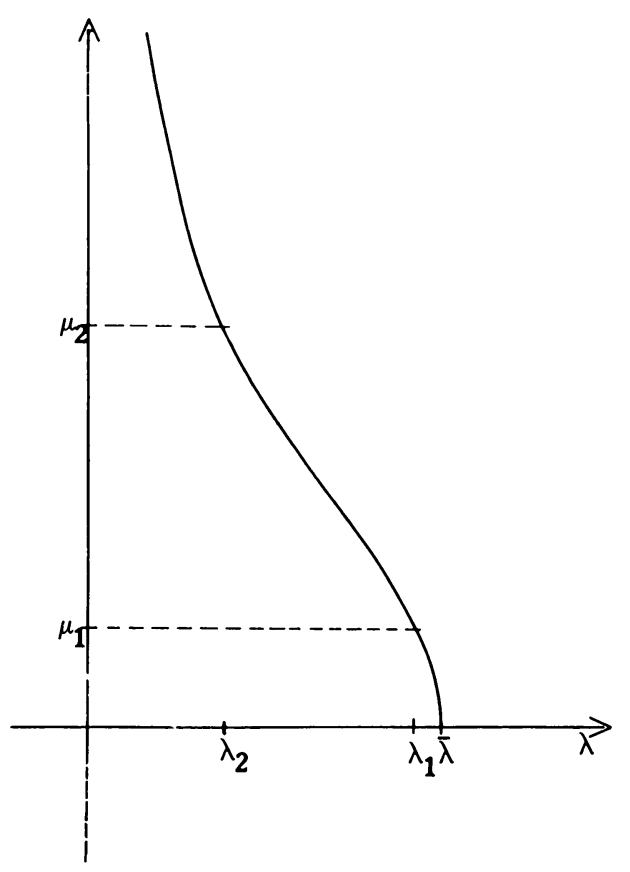

$\operatorname{Graph} \lambda \mapsto \partial_{1} f(m(\lambda), \lambda)$ 
Figure 3.1 shows the set $M . M_{c}=\varnothing$, and only for $m>1 / \chi_{0}$ we have $\partial_{1} f(m, \lambda)$ $>0$ such that all possible bifurcation points are in this region of $M$. The set $\left\{(m, \lambda) \in M \mid m>1 / \chi_{0}\right\}$ can be parametrized over $\lambda$ by $m(\lambda)=h^{-1}((\alpha / \beta) \lambda)$ with $h:] 1 / \chi_{0}, \infty[\rightarrow] 0, \bar{\lambda}\left[, h(m)=m e^{-\chi_{0} m}\right.$. Thus $m(\lambda) \rightarrow+\infty$ for $\lambda \rightarrow 0, m(\lambda)=1 / \chi_{0}$ for $\lambda \rightarrow \bar{\lambda}$. For any $(m, \lambda) \in M$,

$$
\partial_{1} f(m, \lambda)=\alpha \lambda \chi_{0} e^{\chi_{0} m}-\beta=\chi_{0} \beta m-\beta=\beta\left(\chi_{0} m-1\right) .
$$

Thus $\partial_{1} f(m(\lambda), \lambda) \rightarrow+\infty$ for $\lambda \rightarrow 0$ and $\partial_{1} f(m(\lambda), \lambda) \rightarrow 0$ for $\lambda \rightarrow \bar{\lambda}$ with $(d / d \lambda) \partial_{1} f(m(\lambda), \lambda)=\beta \chi_{0} m^{\prime}(\lambda)<0$.

If we assume all eigenvalues $\mu_{j}$ of $v \mapsto \Delta v$ to be simple, then by Theorem 3.1(b)(i) we have infinitely many bifurcation points $\left(m\left(\lambda_{j}\right), \lambda_{j}\right)$ with $\partial_{1} f\left(m\left(\lambda_{j}\right), \lambda_{j}\right)=\mu_{j}$. (See Figures 3.2, 3.3.) More can be said for $n=1$ : In this case we know by (b)(iv) that $C\left(m\left(\lambda_{j}\right), \lambda_{j}\right) \subset O_{j} \times \mathbf{R}^{+}$for all $j \geqslant 1$ with $\partial_{1} f(m, \lambda)=\mu_{j}$ for all $(m, \lambda) \in M$ $\cap C\left(m\left(\lambda_{j}\right), \lambda_{j}\right)$. But $\partial_{1} f(m(\lambda), \lambda)$ is strictly decreasing, so $M \cap C\left(m\left(\lambda_{j}\right), \lambda_{j}\right)=$ $\left\{\left(m\left(\lambda_{j}\right), \lambda_{j}\right)\right\}$. Thus each branch cannot "return" to $M$ and is not relatively compact in $B \times \mathbf{R}^{+}$by (ii). (In fact it is unbounded, as a more detailed treatment shows.)

By $u=\lambda e^{x_{0} v}$ this qualitative bifurcation behaviour also holds for the solution set of the system (2.1), and is illustrated in Figure $3.4(n=1)$. The trivial set $M$ corresponds to $k^{-1}(\{0\})$, the set of constant solutions to (2.1). The spatial pattern of solutions in each of the branches, as indicated in the drawing, holds for both components $u$ and $v$.
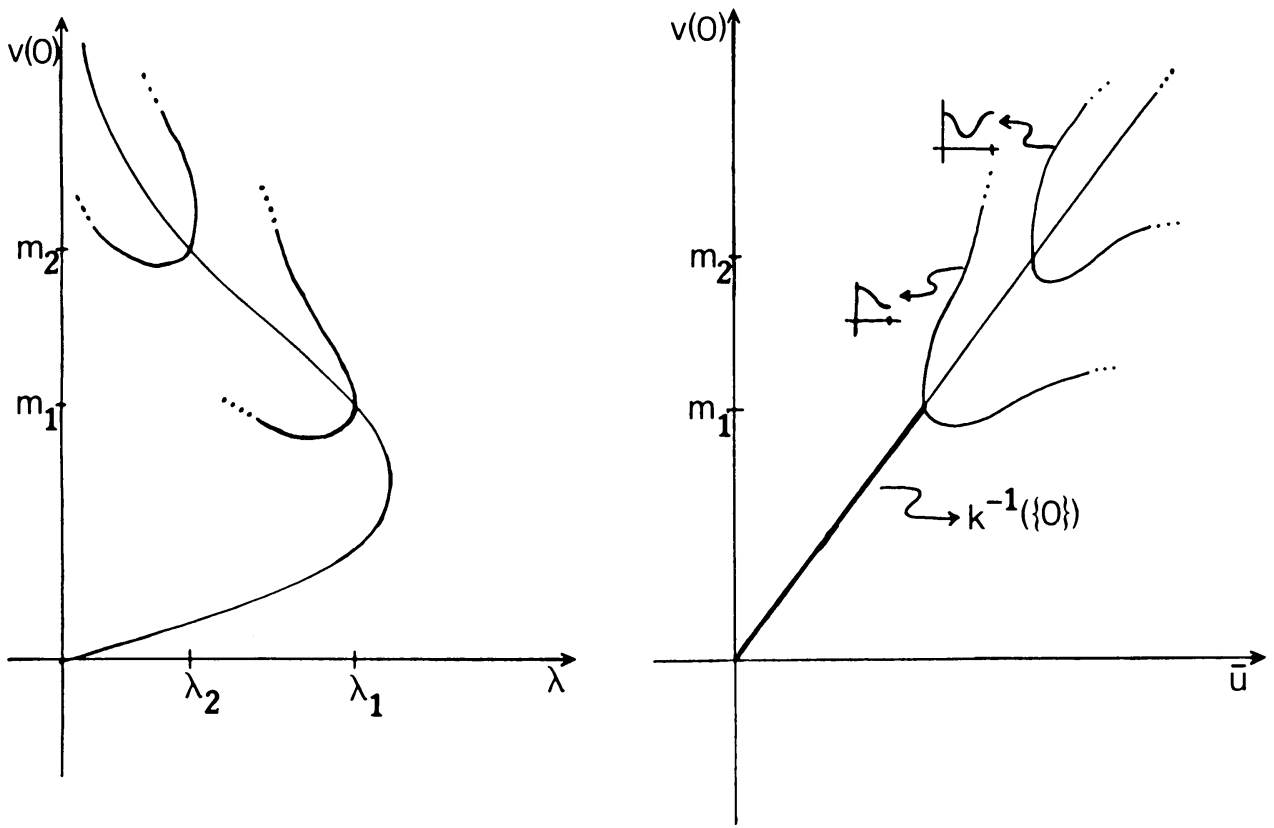

FIGURE 3.3. Bifurcation

FIGURE 3.4 
4. Stability. For the time dependent system

$$
\begin{aligned}
& \partial_{t} u=\nabla \cdot(\mu(u, v) \nabla u-\chi(u, v) \nabla v), \\
& \partial_{t} v=\nu_{0} \Delta v+k(u, v) \\
& \left.\partial_{\nu} u\right|_{\partial \Omega}=\left.\partial_{\nu} v\right|_{\partial \Omega}=0
\end{aligned}
$$

we have

$$
\frac{d}{d t} \int_{\Omega} u(x, t) d t=\int_{\Omega} \partial_{t} u(x, t) d x=0
$$

because of the Neumann conditions. Thus

$$
\bar{u}:=\int_{\Omega} u(x, t) d x
$$

is a constant in time, the total cell mass remains unchanged. A stationary solution thus can only be asymptotically stable with respect to small perturbations $(u, v) \in$ $X_{0} \times X$ with

$$
X_{0}=\left\{u \in X \mid \int_{\Omega} u=0\right\} .
$$

So we will confine ourselves to such perturbations. Also we will see that $\bar{u}$ is the right parameter for exchange of stability in a bifurcation point, that is a supercritical branch with respect to $\bar{u}$ is stable, a subcritical one is unstable (see Figure 4.1). It is also sufficient to prove linearized stability, for the asymptotic stability then follows by a theorem of $\mathbf{H}$. Kielhöfer in [11].

In this section we will assume, for $k$,

$$
\partial_{1} k(r, s) \geqslant 0, \quad \partial_{2} k(r, s)<0 \quad \text { for all }(r, s) \in \mathbf{R}^{+} \times \mathbf{R}^{+} .
$$

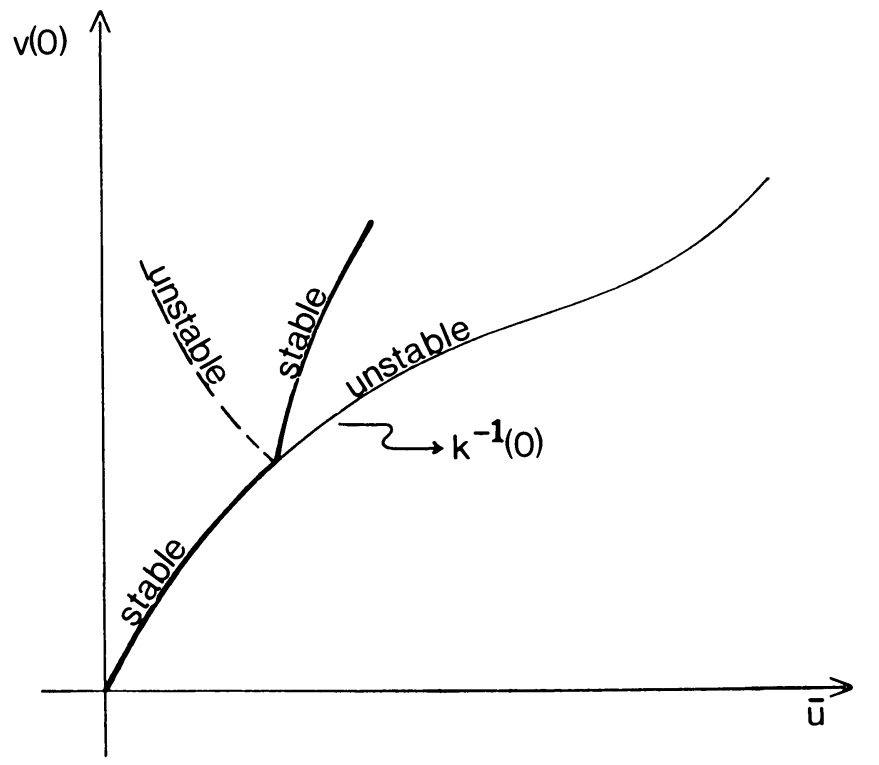

FIGURE 4.1 
This corresponds e.g. to the biological assumptions that the chemoattractant is produced by the cells with a production rate which is a nonincreasing function of $v$, and that the degradation of $v$ increases as $v$ increases. The simplest example for such a mechanism is $k(u, v)=\alpha u-\beta v$. Our first point is the discussion of stability of constant solutions forming the set

$$
N:=\left\{(\sigma, m) \in \mathbf{R}^{+} \times \mathbf{R}^{+} \mid k(\sigma, m)=0\right\} .
$$

The linearization of $G$ around $(\sigma, m) \in N$ is

$$
\left(\begin{array}{c}
\mu \Delta u-\chi \Delta v \\
\nu_{0} \Delta v+k_{1} u+k_{2} v
\end{array}\right),
$$

where we have used the abbreviations

$$
\mu=\mu(\sigma, m), \quad \chi=\chi(\sigma, m), \quad k_{1}=\partial_{1} k(\sigma, m), \quad k_{2}=\partial_{2} k(\sigma, m) .
$$

such that $\mu, \chi, k_{1}, k_{2}$ are constant coefficients.

Let $\left\{\boldsymbol{\omega}_{i} \mid i \in \mathbf{N} \cup\{0\}\right\}$ be a complete orthogonal set (in $L^{2}$ ) of eigenvectors of $v \mapsto \Delta v$ between $X$ and $Y$ with the corresponding eigenvalues

$$
0=\mu_{0}<\mu_{1} \leqslant \mu_{2} \leqslant \cdots, \quad \Delta \omega_{i}+\mu_{i} \omega_{i}=0 .
$$

Testing both equations $D G_{1}(\sigma, m)(u, v)=\eta u, D G_{2}(\sigma, m)(u, v)=\eta v$ with the $\omega_{i}$ we have that $\eta$ is an eigenvalue of $D G(\sigma, m)$ with an eigenvector $(u, v) \in X_{0} \times X$ iff there is an $i \geqslant 0$ such that $\eta$ is an eigenvalue of

$$
A_{i}=\left(\begin{array}{cc}
-\mu \mu_{i}, & \chi \mu_{i} \\
k_{1}, & -\nu_{0} \mu_{i}+k_{2}
\end{array}\right),
$$

with an eigenvector $z \in \mathbf{R}^{+}$for $i \geqslant 1$ and an eigenvector $(0,1)$ for $i=0$. The restriction for $i=0$ follows from $0=\int_{\Omega} u=\left\langle u, \omega_{0}\right\rangle$. Thus $D G(\sigma, m)$ has the following eigenvalues:

$$
\begin{gathered}
\eta_{0}=k_{2} \quad(i=0), \\
\eta_{i}^{+--}=-\frac{1}{2}\left(\nu_{0}+\mu\right) \mu_{i}-k_{2} \\
\quad \pm \sqrt{\left(\left(\nu_{0}+\mu\right) \mu_{i}-k_{2}\right)^{2}-4 \mu \mu_{i}\left(\nu_{0} \mu_{i}-k_{2}\right)+4 \chi \mu_{i} k_{1}} \quad(i \geqslant 1) .
\end{gathered}
$$

All eigenvalues are real because of the expression under the radical sign being equal to $\left(\left(\mu-\nu_{0}\right) \mu_{i}+k_{2}\right)^{2}+4 \chi \mu_{i} k_{1}$ which is positive by (4.3). Also $k_{2}<0$ and $-\left(\left(\nu_{0}+\mu\right) \mu_{i}-k_{2}\right)<0$, such that all eigenvalues of $D G(\sigma, m)$ are negative iff

$$
4 \chi \mu_{i} k_{1}-4 \mu \mu_{i}\left(\nu_{0} \mu_{i}-k_{2}\right)<0 \text { for all } i \geqslant 1 \text {, }
$$

i.e., $\chi k_{1}+\mu k_{2}<\mu \nu_{0} \mu_{i}$ for all $i \geqslant 1$, or equivalently

$$
\frac{1}{\nu_{0}}\left(\frac{\chi}{\mu} k_{1}+k_{2}\right)<\mu_{1} \text {. }
$$

Conversely, if

$$
\frac{1}{\nu_{0}}\left(\frac{\chi}{\mu} k_{1}+k_{2}\right)>\mu_{1}
$$

then one of the eigenvalues $\eta_{1}^{+,-}$is positive and $(\sigma, m)$ is unstable. 
For (4.4) to imply stability one has to show that 0 is not a limit point of the eigenvalues (see [11]). This follows from

$$
\begin{aligned}
\eta_{i}^{+,-} & \leqslant \frac{1}{2}-a_{i}+\sqrt{a_{i}^{2}-b_{i}} \leqslant \frac{1}{2}-a_{i}+a_{i}-\frac{b_{i}}{2} \\
& =\frac{-b_{i}}{2} \leqslant-\frac{b_{1}}{4}<0 \quad \text { by }(4.4)
\end{aligned}
$$

with $a_{i}=\left(\nu_{0}+\mu\right) \mu_{i}-k_{2}, b_{i}=4 \mu \mu_{i}\left(\nu_{0} \mu_{i}-k_{2}\right)-4 \chi \mu_{i} k_{1}$.

By $\sigma=(m, \lambda)$ and $\partial_{1}(m, \lambda)=\chi / \mu$ (see $\left.\S 2\right)$ we can translate (4.4), (4.5) into conditions on $f(m, \lambda)=\left(1 / \nu_{0}\right) k(\varphi(m, \lambda), m)$.

4.1 TheOREM. Let $(\sigma, m) \in N$ be a constant solution of $G(u, v)=0$, i.e. $\sigma=$ $\varphi(m, \lambda)$ for some $\lambda>0$. Then $(\sigma, m)$ is asymptotically stable with respect to small perturbations $(u, v) \in X_{0} \times X$ if $\partial_{1} f(m, \lambda)<\mu_{1}$. If $\partial_{1} f(m, \lambda)>\mu_{2}$, then $(\sigma, m)$ is unstable.

Proof. With $\sigma=(m, \lambda), \partial_{1} \varphi(m, \lambda)=\chi / \mu, f(m, \lambda)=\left(1 / \nu_{0}\right) k(\varphi(m, \lambda), m)$, we have

$$
\begin{aligned}
\partial_{1} f(m, \lambda) & =\frac{1}{\nu_{0}}\left(\partial_{1} k(\varphi(m, \lambda), m) \partial_{1} \varphi(m, \lambda)+\partial_{2} k(\varphi(m, \lambda), m)\right) \\
& =\frac{1}{\nu_{0}}\left(k_{1} \frac{\chi}{\mu}+k_{2}\right) .
\end{aligned}
$$

So by above calculations resulting in (4.4), (4.5) the theorem is proved. Theorem 4.1 applied to the Example 3.2 gives that all points in $N=k^{-1}(\{0\})$ with $m<m_{1}$ are stable. This region has been marked in Figure 3.4.

For exchange of stability we make use of the following theorem by Crandall and Rabinowitz [7]:

4.2 Theorem (CANDAll, Rabinowitz). Let $Z_{1}, Z_{2}$ be real Banach spaces, $V \in Z_{1}$ an open neighbourhood of $0, \mathscr{F}=(a, b) \subset \mathbf{R}$ an open interval, and $F: V \times \mathscr{F} \rightarrow Z_{2} a k$ times continuously Frechét differentiable mapping. Suppose that $\sigma_{0} \in \mathscr{F}$ and that

(i) $F(0, \sigma)=0$ for all $\sigma \in \mathscr{F}$,

(ii) $\operatorname{dim} N\left(F_{z}\left(0, \sigma_{0}\right)\right)=\operatorname{codim} R\left(F_{z}\left(0, \sigma_{0}\right)\right)=1$,

(iii) $F_{\sigma z}\left(0, \sigma_{0}\right) z_{0} \notin R\left(F_{z}\left(0, \sigma_{0}\right)\right)$, where $z_{0}$ spans $N\left(F_{z}\left(0, \sigma_{0}\right)\right)$,

(iv) $z_{0} \notin R\left(F_{z}\left(0, \sigma_{0}\right)\right)$ ( 0 is an algebraically simple eigenvalue of $\left.F_{z}\left(0, \sigma_{0}\right)\right)$.

Then

(a)There exists $\varepsilon_{0}>0$ and $(k-1)$-times continuously differentiable functions $\sigma$ : ]$-\varepsilon_{0}, \varepsilon_{0}[\rightarrow \mathscr{F}, z:]-\varepsilon_{0}, \varepsilon_{0}\left[\rightarrow Z_{1}\right.$ such that $\sigma(0)=\sigma_{0}, z(0)=0, z^{\prime}(0)=z_{0}$ and

$$
F(z(\varepsilon), \sigma(\varepsilon))=0 \quad \text { for all } \varepsilon \in]-\varepsilon_{0}, \varepsilon_{0}[\text {. }
$$

$F^{-1}(\{0\})$ near $\left(0, \sigma_{0}\right)$ consists precisely of the curves $(0, \sigma)$ and $(z(\varepsilon), \sigma(\varepsilon))$.

(b) There exist continuously differentiable functions of eigenvalues

$$
\eta:] \sigma_{0}-\varepsilon_{0}, \sigma_{0}+\varepsilon_{0}[\rightarrow \mathbf{R}, \quad \gamma:]-\varepsilon_{0}, \varepsilon_{0}[\rightarrow \mathbf{R}
$$

such that

$\eta(\sigma)$ is an isolated simple eigenvalue of $F_{z}(0, \sigma)$, 
$\gamma(\varepsilon)$ is an isolated simple eigenvalue of $F_{z}(z(\varepsilon), \sigma(\varepsilon))$ with $\eta\left(\sigma_{0}\right)=\gamma(0)=0$ and $\eta^{\prime}\left(\sigma_{0}\right) \neq 0$. Moreover,

$$
\operatorname{sign} \gamma(\varepsilon)=\operatorname{sign}\left(-\varepsilon \sigma^{\prime}(\varepsilon) \eta^{\prime}\left(\sigma_{0}\right)\right) .
$$

(We have used the notations $F_{\sigma}, F_{z}, F_{\sigma z}$, etc. for the various derivatives of $F$ with respect to $\sigma \in \mathscr{F}, z \in Z_{1} . N(A)$, resp. $R(A)$, denotes the null space, resp. the range of a linear mapping A.)

This theorem can be used to calculate exchange of stability near the bifurcation point $\left(0, \sigma_{0}\right)$. Suppose all eigenvalues of $F_{z}\left(0, \sigma_{0}\right)$ are negative for $\sigma<\sigma_{0}$. Then $\eta(\sigma)$ as well as $\gamma(\varepsilon)$ have to be the largest eigenvalues of $F_{z}(0, \sigma)$ and $F_{z}(z(\varepsilon), \sigma(\varepsilon)$ ) (see [9]). In this situation the sign of $\gamma(\varepsilon)$ is the same as $-\varepsilon \sigma^{\prime}(\varepsilon)$. So bifurcating solutions are stable if the branch turns to the right with respect to $\sigma$; they are unstable if it turns left.

We will use this theorem for $G$ by making a change of variables for which $\sigma=\bar{u}$ becomes the parameter and $Z_{1}=X_{0} \times X$. Since we have assumed $\partial_{1} k \geqslant 0, \partial_{2} k<0$ the set $N=\{(\sigma, m) \mid k(\sigma, m)=0\}$ can be parametrized over $\sigma$ by some $C^{2}$-function $\psi: D(\psi) \mathbf{R}^{+} \rightarrow \mathbf{R}^{+}:$

$$
N=\{(\sigma, \psi(\sigma)) \mid \sigma \in D(\psi)\} .
$$

4.3 THEOREM. Let $\mu, \chi, k$ be three times continuously differentiable, and let $\left(\sigma_{0}, m_{0}\right)$ $\in N$ be a bifurcation point of $G$ with $\sigma_{0}=\varphi\left(m_{0}, \lambda_{0}\right),\left(m_{0}, \lambda_{0}\right) \in M$ being $a$ bifurcation point of (3.1) satisfying the assumptions of Theorem 3.1(b) with $j=1$, i.e., $\partial_{1} f\left(m_{0}, \lambda_{0}\right)=\mu_{1}$, yielding a parametrization $\lambda \mapsto m(\lambda), m\left(\lambda_{0}\right)=m_{0}$, of $M$ near $\left(m_{0}, \lambda_{0}\right)$, and

$$
c\left(\lambda_{0}\right):=\left.\frac{d}{d \lambda} \partial_{1} f(m(\lambda), \lambda)\right|_{\lambda=\lambda_{0}} \neq 0 .
$$

Then there is a twice continuously differentiable branch of nonhomogeneous solutions

$$
\varepsilon \mapsto(U(\varepsilon), V(\varepsilon)), \quad \varepsilon \in]-\varepsilon_{0}, \varepsilon_{0}[
$$

with $G(U(\varepsilon), V(\varepsilon))=0,(U(0), V(0))=\left(\sigma_{0}, m_{0}\right), V^{\prime}(0)=\psi^{\prime}\left(\sigma_{0}\right) \int_{\Omega} U^{\prime}(0)$.

Near $\left(\sigma_{0}, m_{0}\right)$ the set $G^{-1}(\{0\})$ consists of $N$ and the points $(U(\varepsilon), V(\varepsilon))$.

Let $\eta(\sigma)$ be the largest eigenvalue of $\left.D G(\sigma, \psi(\sigma))\right|_{X_{0} \times X}$. Then $\eta(\sigma)$ is simple, $\eta\left(\sigma_{0}\right)=0, \eta^{\prime}\left(\sigma_{0}\right) \neq 0$ and the solutions $(U(\varepsilon), V(\varepsilon))$ are

stable, if $\left(\varepsilon \frac{d}{d \lambda} \int_{\Omega} U(\varepsilon)\right) \eta^{\prime}\left(\sigma_{0}\right)<0 \quad$ (supercritical bifurcation),

unstable, if $\left(\varepsilon \frac{d}{d \lambda} \int_{\Omega} U(\varepsilon)\right) \eta^{\prime}\left(\sigma_{0}\right)>0 \quad$ (subcritical bifurcation).

Proof. The change of variables

$$
(u, v, \sigma) \mapsto(\sigma+u, \psi(\sigma)+v)
$$

is a $C^{3}$-diffeomorphism between a neighbourhood $V \times \mathscr{F} \subset X_{0} \times X \times \mathbf{R}^{+}$of $\left(0, \sigma_{0}\right)$ and a neighbourhood $U \subset X \times X$ of $\left(\sigma_{0}, m_{0}\right)$. We will apply Theorem 4.2 to

$$
F: V \times \mathscr{F} \rightarrow Y_{0} \times Y, \quad F(u, v, \sigma)=G(\sigma+u, \psi(\sigma)+v),
$$

where $Y_{0}=\left\{u \in Y \mid \int_{\Omega} u=0\right\}$. 
(i) then is satisfied since $F(0,0, \sigma)=G(\sigma, \psi(\sigma))=0$. For (ii) we observe $F_{(u, v)}\left(0,0, \sigma_{0}\right)=\left.D G\left(\sigma_{0}, m_{0}\right)\right|_{X_{0} \times X}: X_{0} \times X \rightarrow Y_{0} \times Y$. By Theorem 2.2, $D G\left(\sigma_{0}, m_{0}\right)(u, v)=0$ iff

$$
u=\partial_{1} \varphi\left(m_{0}, \lambda_{0}\right) v+\lambda \partial_{2} \varphi\left(m_{0}, \lambda_{0}\right)
$$

for some $\lambda \in \mathbf{R}$ and

$$
v+\partial_{1} f\left(m_{0}, \lambda_{0}\right) v+\lambda \partial_{2} f\left(m_{0}, \lambda_{0}\right)=0
$$

where (4.7) also reads

$$
\nu_{0} \Delta v+\partial_{1} k\left(\sigma_{0}, m_{0}\right)\left(\partial_{1} \varphi\left(m_{0}, \lambda_{0}\right) v+\lambda \partial_{2} \varphi\left(m_{0}, \lambda_{0}\right)\right)+\partial_{2} k\left(\sigma_{0}, m_{0}\right) v=0
$$

because of the definition of $f(2.17)$ and since $\sigma_{0}=\left(m_{0}, \lambda_{0}\right)$. If we restrict $u$ to $X_{0}$, (4.6) and (4.8) yields $\int_{\Omega} v=0$, and thus $\lambda=0$ using (4.6) again. Thus $u=$ $\partial_{1} \varphi\left(m_{0}, \lambda_{0}\right) v, \int v=0$, and

$$
\Delta v+\partial_{1} f\left(m_{0}, \lambda_{0}\right) v=0 .
$$

Now $\partial_{1} f\left(m_{0}, \lambda_{0}\right)=\mu_{1}$, thus $v \in \operatorname{span}\left\{\omega_{1}\right\}$. As a result,

$$
N\left(F_{(u, v)}\left(0,0, \sigma_{0}\right)\right)=\operatorname{span}\left\{\left(\partial_{1} \varphi\left(m_{0}, \lambda_{0}\right) \omega_{1}, \omega_{1}\right)\right\} \text {. }
$$

In order to show that $F_{(u, v)}\left(0,0, \sigma_{0}\right)$ is Fredholm of index 0 , we first show the corresponding property for $D G\left(\sigma_{0}, m_{0}\right): X \times X \rightarrow Y \times Y$ : With

$$
\begin{aligned}
\tilde{\mu}(\sigma) & =\mu(\sigma, \psi(\sigma)), \quad \tilde{\chi}(\sigma)=\chi(\sigma, \psi(\sigma)), \\
k_{1}(\sigma) & =\partial_{1} k(\sigma, \psi(\sigma)), \quad k_{2}(\sigma)=\partial_{2} k(\sigma, \psi(\sigma))
\end{aligned}
$$

we have

$$
D G(\sigma, \psi(\sigma))(u, v)=\left(\begin{array}{c}
\tilde{\mu}(\sigma) \Delta u-\tilde{\chi}(\sigma) \Delta v \\
\nu_{0} \Delta v+k_{1}(\sigma) u+k_{2}(\sigma) v
\end{array}\right)
$$

The matrix

$$
A=\left(\begin{array}{cc}
\tilde{\mu}\left(\sigma_{0}\right), & \tilde{\chi}\left(\sigma_{0}\right) \\
0, & \nu_{0}
\end{array}\right)
$$

is invertible.

Let $K\left(w_{1}, w_{2}\right)=\left(L w_{1}, L w_{2}\right)$, where $L: Y \rightarrow X$ is the inverse of $\Delta$-id. Then $K \circ A^{-1} D G\left(\sigma_{0}, m_{0}\right): X \times X \rightarrow X \times X$ is a compact perturbation of identity:

$$
K \circ A^{-1} D G\left(\sigma_{0}, m_{0}\right)(u, v)=\left(\begin{array}{l}
u \\
v
\end{array}\right)+K\left[\left(\begin{array}{l}
u \\
v
\end{array}\right)+A^{-1}\left(\begin{array}{c}
0 \\
k_{1}\left(\sigma_{0}\right) u+k_{2}\left(\sigma_{0}\right) v
\end{array}\right)\right] .
$$

Since the expression in the square brackets is in $X \times X$ and the embedding $X \times X \rightarrow Y \times Y$ is compact, we have that $K \circ A^{-1} D G\left(\sigma_{0}, m_{0}\right)$-id is compact. Thus $D G\left(\sigma_{0}, m_{0}\right): X \times X \rightarrow Y \times Y$ is Fredholm of index 0 . The same is true for $F_{(u, v)}\left(0,0, \sigma_{0}\right): X_{0} \times X \rightarrow Y_{0} \times Y$ since $\left(1, \psi^{\prime}\left(\sigma_{0}\right)\right)$ is in the nullspace of $D G\left(\sigma_{0}, m_{0}\right)$ and is complementary to $X_{0} \times X$.

Proof of (iii). By (4.10)

$$
\begin{aligned}
F_{\sigma(u, v)}\left(0, \sigma_{0}\right)(u, v) & =\left.\frac{d}{d \sigma}\left(F_{(u, v)}(0, \sigma)(u, v)\right)\right|_{\sigma=\sigma_{0}} \\
& =\left(\begin{array}{c}
\tilde{\mu}^{\prime}\left(\sigma_{0}\right) \Delta u-\tilde{\chi}^{\prime}\left(\sigma_{0}\right) \Delta v \\
k_{1}^{\prime}\left(\sigma_{0}\right) u+k_{2}^{\prime}\left(\sigma_{0}\right) v
\end{array}\right) .
\end{aligned}
$$


We have to show (see 4.9) that $F_{\sigma(u, v)}\left(0, \sigma_{0}\right)\left(\partial_{1} \varphi\left(m_{0}, \lambda_{0}\right) \omega_{1}, \omega_{2}\right)$ is not in $R\left(F_{\left(u, v^{\prime}\right)}\left(0, \sigma_{0}\right)\right) . \partial_{1} \varphi\left(m_{0}, \lambda_{0}\right)=\tilde{\chi}\left(\sigma_{0}\right) / \tilde{\mu}\left(\sigma_{0}\right)$. Thus

$$
\begin{aligned}
& F_{\sigma\left(u, v^{\prime}\right)}\left(0, \sigma_{0}\right)\left(\tilde{\chi}\left(\sigma_{0}\right) \omega_{1}, \tilde{\mu}\left(\sigma_{0}\right) \omega_{1}\right) \\
& =\left(\begin{array}{c}
-\mu_{1} \omega_{1}\left(\tilde{\mu}^{\prime}\left(\sigma_{0}\right) \tilde{\chi}\left(\sigma_{0}\right)-\tilde{\mu}\left(\sigma_{0}\right) \tilde{\chi}^{\prime}\left(\sigma_{0}\right)\right) \\
\omega_{1}\left(k_{1}^{\prime}\left(\sigma_{0}\right) \tilde{\chi}\left(\sigma_{0}\right)+\tilde{\mu}\left(\sigma_{0}\right) k_{2}^{\prime}\left(\sigma_{0}\right)\right)
\end{array}\right) .
\end{aligned}
$$

Assuming this to be in $R\left(F_{(u, v)}\left(0, \sigma_{0}\right)\right)$ we get by testing with $\omega_{1}$ :

$$
\left(\begin{array}{c}
-\mu_{1}\left(\tilde{\mu}^{\prime}\left(\sigma_{0}\right) \tilde{\chi}\left(\sigma_{0}\right)-\tilde{\mu}\left(\sigma_{0}\right) \tilde{\chi}^{\prime}\left(\sigma_{0}\right)\right) \\
k_{1}^{\prime}\left(\sigma_{0}\right) \tilde{\chi}\left(\sigma_{0}\right)+\tilde{\mu}\left(\sigma_{0}\right) k_{2}^{\prime}\left(\sigma_{0}\right)
\end{array}\right) \in R\left(A_{1}\right)
$$

with

$$
A_{1}=\left(\begin{array}{cc}
-\tilde{\mu}\left(\sigma_{0}\right) \mu_{1}, & \tilde{\chi}\left(\sigma_{0}\right) \mu_{1} \\
k_{1}\left(\sigma_{0}\right), & -\nu_{0} \mu_{1}+k_{2}\left(\sigma_{0}\right)
\end{array}\right) .
$$

By $\mu_{1}=\partial_{1} f\left(m_{0}, \lambda_{0}\right)=\left(1 / \nu_{0}\right)\left(k_{1}\left(\sigma_{0}\right) \tilde{\chi}\left(\sigma_{0}\right) / \tilde{\mu}\left(\sigma_{0}\right)+k_{2}\left(\sigma_{0}\right)\right)$ one can easily calculate that $\operatorname{det} A_{2}=0$ and $R\left(A_{1}\right)=\operatorname{span}\left\{\left(1,-k_{1}\left(\sigma_{0}\right) / \tilde{\mu}\left(\sigma_{0}\right) \mu_{1}\right)^{\tau}\right\}$. Thus our assumption would give

$$
k_{1}^{\prime}\left(\sigma_{0}\right) \tilde{\chi}\left(\sigma_{0}\right)+\tilde{\mu}\left(\sigma_{0}\right) k_{2}^{\prime}\left(\sigma_{0}\right)=\left(k_{1}\left(\sigma_{0}\right) / \tilde{\mu}\left(\sigma_{0}\right)\right)\left(\tilde{\mu}^{\prime}\left(\sigma_{0}\right) \tilde{\chi}\left(\sigma_{0}\right)-\tilde{\mu}\left(\sigma_{0}\right) \tilde{\chi}^{\prime}\left(\sigma_{0}\right)\right),
$$
i.e.,

$$
k_{1}^{\prime}\left(\sigma_{0}\right) \frac{\tilde{\chi}\left(\sigma_{0}\right)}{\tilde{\mu}\left(\sigma_{0}\right)}+k_{1}\left(\sigma_{0}\right)\left(\frac{d}{d \sigma} \frac{\tilde{\chi}\left(\sigma_{0}\right)}{\tilde{\mu}\left(\sigma_{0}\right)}\right)+k_{2}^{\prime}\left(\sigma_{0}\right)=0 .
$$

This is a contradiction to $\left.(d / d \lambda) \partial_{1} f(m(\lambda), \lambda)\right|_{\lambda=\lambda_{0}} \neq 0$ : With $\sigma(\lambda)=\varphi(m(\lambda), \lambda)$ we get

$$
\nu_{0} \partial_{1} f(m(\lambda), \lambda)=k_{1}(\sigma(\lambda)) \tilde{\chi}(\lambda(\sigma)) / \tilde{\mu}(\sigma(\lambda))+k_{2}(\sigma(\lambda))
$$

so

$$
\begin{aligned}
& \left.\nu_{0} \frac{d}{d \lambda} \partial_{1} f(m(\lambda), \lambda)\right|_{\lambda=\lambda_{0}} \\
& \quad=\left[k_{1}^{\prime}\left(\sigma_{0}\right) \frac{\tilde{\chi}\left(\sigma_{0}\right)}{\tilde{\mu}\left(\sigma_{0}\right)}+k_{1}\left(\sigma_{0}\right)\left(\frac{d}{d \sigma} \frac{\tilde{\chi}\left(\sigma_{0}\right)}{\tilde{\mu}\left(\sigma_{0}\right)}\right)+k_{2}^{\prime}\left(\sigma_{0}\right)\right] \sigma^{\prime}\left(\lambda_{0}\right) .
\end{aligned}
$$

This proves (iii). In the same way we get property (iv), namely $\left(\tilde{\chi}\left(\sigma_{0}\right) \omega_{1}, \tilde{\mu}\left(\sigma_{0}\right) \omega_{1}\right)$ $\notin R\left(F_{(u, v)}\left(0, \sigma_{0}\right)\right)$, since otherwise $\left(\tilde{\chi}\left(\sigma_{0}\right), \tilde{\mu}\left(\sigma_{0}\right)\right)$ would be in

$$
R\left(A_{1}\right)=\operatorname{span}\left\{\left(1,-\frac{k_{1}\left(\sigma_{0}\right)}{\tilde{\mu}\left(\sigma_{0}\right) \mu_{1}}\right)^{\tau}\right\}
$$

in contradiction to $k_{1} \geqslant 0$. By Theorem 4.2 we obtain a bifurcating branch

$$
\varepsilon \mapsto(u(\varepsilon), v(\varepsilon), \sigma(\varepsilon)),
$$

thus

$$
(U(\varepsilon), V(\varepsilon))=(u(\varepsilon)+\sigma(\varepsilon), v(\varepsilon)+\psi(\sigma(\varepsilon)))
$$

gives the branch in the theorem's assertion.

$$
V^{\prime}(0)=v^{\prime}(0)+\psi^{\prime}\left(\sigma_{0}\right) \sigma^{\prime}(0)=\omega_{1}+\psi^{\prime}\left(\sigma_{0}\right) \int_{\Omega} U^{\prime}(0) .
$$


The sign of the largest eigenvalue $\gamma(\varepsilon)$ of $D G(U(\varepsilon), V(\varepsilon))$ with respect to eigenvectors in $X_{0} \times X$ can be computed by Theorem 4.2:

$$
\begin{aligned}
\operatorname{sign} \gamma(\varepsilon) & =\operatorname{sign}\left(-\varepsilon \sigma^{\prime}(\varepsilon) \eta^{\prime}\left(\sigma_{0}\right)\right) \\
& =\operatorname{sign}\left(-\varepsilon\left(\frac{d}{d \varepsilon} \int_{\Omega} U(\varepsilon)\right) \eta^{\prime}\left(\sigma_{0}\right)\right) .
\end{aligned}
$$

Thus the theorem is proved and we get the picture of subcritical or supercritical bifurcation and exchange of stability as indicated in Figure 4.1.

The sign of $\eta^{\prime}\left(\sigma_{0}\right)$ can be calculated by $\partial_{1} f(m(\lambda), \lambda)$ for $\lambda$ near $\lambda_{0}$, since $\partial_{1} f(m(\lambda), \lambda)>\mu_{1}$ or $<\mu_{1}$ determines the sign of the largest eigenvalue of $D G(\varphi(m(\lambda), \lambda), m(\lambda)$ ) (Theorem 4.1). $\sigma$ depends on $\lambda$ by $\sigma(\lambda)=\varphi(m(\lambda), \lambda)$. Thus

$$
\sigma^{\prime}\left(\lambda_{0}\right)=\partial_{1} \varphi\left(m\left(\lambda_{0}\right), \lambda_{0}\right) m^{\prime}\left(\lambda_{0}\right)+\partial_{2} \varphi\left(m\left(\lambda_{0}\right), \lambda_{0}\right) .
$$

Because of $f(m(\lambda), \lambda)=0$ we get

$$
m^{\prime}(\lambda)=-\partial_{2} f(m(\lambda), \lambda) / \partial_{1} f(m(\lambda), \lambda)
$$

Using $\partial_{1} f\left(m\left(\lambda_{0}\right), \lambda_{0}\right)=\mu_{1}$ we arrive at (arguments omitted):

$$
\begin{aligned}
\sigma^{\prime}\left(\lambda_{0}\right) & =-\partial_{1} \varphi \frac{\partial_{2} f}{\mu_{1}}+\partial_{2} \varphi=-\partial_{1} \varphi \frac{\partial_{1} k \partial_{2} \varphi}{\mu_{1}}+\partial_{2} \varphi \\
& =\frac{\partial_{2} \varphi}{\mu_{1}}\left(-\partial_{1} f+\partial_{2} k+\mu_{1}\right)=\frac{\partial_{2} \varphi \partial_{2} k}{\mu_{1}}<0
\end{aligned}
$$

because of assumption (4.3). Since

$$
\begin{aligned}
\operatorname{sign}\left(\eta^{\prime}\left(\sigma_{0}\right) \sigma^{\prime}\left(\lambda_{0}\right)\right) & =\operatorname{sign}\left(\left.\frac{d}{d \lambda} \eta(\sigma(\lambda))\right|_{\lambda=\lambda_{0}}\right) \\
& =\operatorname{sign}\left(\left.\frac{d}{d \lambda} \partial_{1} f(m(\lambda), \lambda)\right|_{\lambda=\lambda_{0}}\right),
\end{aligned}
$$

this results in sign $\eta^{\prime}\left(\sigma_{0}\right)=-\operatorname{sign} c\left(\lambda_{0}\right)$ with $c\left(\lambda_{0}\right)=\left.(d / d \lambda) \partial_{1} f(m(\lambda), \lambda)\right|_{\lambda=\lambda_{0}}$. So we get

4.4 Corollary. Let all assumptions of Theorem 4.3 be satisfied. Then for the branch $\varepsilon \rightarrow(U(\varepsilon), V(\varepsilon))$ of that theorem, $(U(\varepsilon), V(\varepsilon))$ is

$$
\begin{gathered}
\text { stable, } \quad \text { if } \varepsilon\left(\frac{d}{d \varepsilon} \int_{\Omega} U(\varepsilon)\right) c\left(\lambda_{0}\right)<0, \\
\text { unstable, } \quad \text { if } \varepsilon\left(\frac{d}{d \varepsilon} \int_{\Omega} U(\varepsilon)\right) c\left(\lambda_{0}\right)>0,
\end{gathered}
$$

where $c\left(\lambda_{0}\right)=\left.(d / d \lambda) \partial_{1} f(m(\lambda), \lambda)\right|_{\lambda=\lambda_{0}}$.

For space dimension $n=1$ we can show that the bifurcation is one-sided, and that the direction of the bifurcation branch is determined by an algebraic expression involving derivatives up to order three of $\varphi$ and $f$ at the bifurcation point $\left(m_{0}, \lambda_{0}\right)$ : 
4.5 THEOREM (Direction OF THE BIFURCATING BRANCH). Let all assumptions of Theorem 4.3 be satisfied, let $n=1$, and assume that all nonlinearities are four times continuously differentiable. Define

$$
\begin{aligned}
J\left(m_{0}, \lambda_{0}\right):= & c\left(\lambda_{0}\right) \cdot\left(\partial_{1}^{2} \varphi \partial_{1} f-\partial_{1} \varphi \partial_{1}^{2} f\right)\left(m_{0}, \lambda_{0}\right) \\
& +\frac{1}{6} d\left(\lambda_{0}\right) \cdot\left(5\left(\partial_{1}^{2} f\right)^{2}-3 \partial_{1} f \partial_{1}^{3} f\right)\left(m_{0}, \lambda_{0}\right),
\end{aligned}
$$

where

$$
c\left(\lambda_{0}\right)=\frac{d}{d \lambda} \partial_{1} f(m(\lambda), \lambda)_{\lambda=\lambda_{0}}, \quad d\left(\lambda_{0}\right)=\left.\frac{d}{d \lambda} \varphi(m(\lambda), \lambda)\right|_{\lambda=\lambda_{0}} .
$$

Then for small $\varepsilon$, the bifurcating solutions $(U(\varepsilon), V(\varepsilon))$ are

$$
\begin{array}{cl}
\text { stable, } & \text { if } J\left(m_{0}, \lambda_{0}\right)<0, \\
\text { unstable, } & \text { if } J\left(m_{0}, \lambda_{0}\right)>0 .
\end{array}
$$

Proof. Solutions $(U(\varepsilon), V(\varepsilon))$ correspond to solutions $(V(\varepsilon), \Lambda(\varepsilon))$ of

$$
v^{\prime \prime}+f(v, \lambda)=0
$$

with $V(0)=m_{0}, \Lambda(0)=\lambda_{0}, V$ and $\Lambda$ are three times continuously differentiable.

By differentiating (4.11) three times with respect to $\varepsilon$ we get equations for

$$
\begin{array}{ll}
v_{1}:=V^{\prime}(0), & v_{2}:=V^{\prime \prime}(0), \quad v_{3}:=V^{\prime \prime \prime}(0), \\
\lambda_{1}:=\Lambda^{\prime}(0), & \lambda_{2}:=\Lambda^{\prime \prime}(0), \quad \lambda_{3}:=\Lambda^{\prime \prime \prime}(0),
\end{array}
$$

which can be solved for $v_{1}, v_{2}, \lambda_{1}, \lambda_{2}$ in one space dimension. The bifurcation of (4.11) has to be one-sided, since for fixed $\lambda$ there have to be always two solutions (reflection in a max or min). Thus necessarily $\lambda_{1}=\Lambda^{\prime}(0)=0$. So we get the three equations (observing $\partial_{1} f\left(m_{0}, \lambda_{0}\right)=\mu_{1}$ and omitting arguments $\left(m_{0}, \lambda_{0}\right)$ ):

$$
\begin{gathered}
v_{1}^{\prime \prime}+\mu_{1} v_{1}=0, \\
v_{2}^{\prime \prime}+\mu_{1} v_{2}+\partial_{1}^{2} f v_{1}^{2}+\partial_{2} f \lambda_{2}=0, \\
v_{3}^{\prime \prime}+\mu_{1} v_{3}+3 \partial_{1}^{2} f v_{1} v_{2}+\partial_{1}^{3} f v_{1}^{3}+3 \partial_{1} \partial_{2} f v_{1} \lambda_{2}+\partial_{2} f \lambda_{3}=0 .
\end{gathered}
$$

Thus by (4.12), $v_{1}=\alpha \omega_{1}$ for some $\alpha$ in $\mathbf{R}$. By Theorem 4.3 we also have $V^{\prime}(0)=\omega_{1}$ $+\psi^{\prime}\left(\sigma_{0}\right) \int_{\Omega} U^{\prime}(0)$. This means

$$
v_{1}=\omega_{1}=\sqrt{2} \cos \pi x \text { and } \int_{\Omega} U^{\prime}(0)=0 .
$$

(Without loss of generality we can take $\Omega=] 0,1\left[, \omega_{1}=\sqrt{2} \cos \pi x\right.$.) As can be easily calculated,

$$
\bar{v}_{2}=\left(1 / \mu_{1}\right)\left[\frac{1}{3} \partial_{1}^{2} f \omega_{1}^{2}-\frac{4}{3} \partial_{1}^{2} f-\partial_{2} f \lambda_{2}\right]
$$

is a special solution to (4.13),

$$
v_{2}=\alpha \omega_{1}+\bar{v}_{2}
$$

thus being the general solution. $\lambda_{2}$ can be calculated from (4.14): $v^{\prime \prime}+\mu_{1} v$ is a symmetric operator with nullspace $\operatorname{span}\left\{\omega_{1}\right\}$. Thus necessarily

$$
0=\int_{\Omega} 3 \partial_{1}^{2} f \omega_{1}^{2} v_{2}+\partial_{1}^{3} f \omega_{1}+3 \partial_{1} \partial_{2} f \omega_{1}^{3} \lambda_{2}+\partial_{2} f \lambda_{3} \omega_{1} .
$$

By (4.16), (4.17) and using

$$
\int_{\Omega} \omega_{1}=0, \quad \int \omega_{1}^{2}=1, \quad \int \omega_{1}^{3}=0, \quad \int \omega_{1}^{4}=\frac{3}{2},
$$


this gives

$$
0=3 \lambda_{2}\left(\partial_{1} \partial_{2} f-\frac{\partial_{1}^{2} f \partial_{2} f}{\mu_{1}}\right)-\frac{5}{2 \mu_{1}}\left(\partial_{1}^{2} f\right)^{2}+\frac{3}{2} \partial_{1}^{3} f
$$

where the factor of $3 \lambda_{2}$ can be calculated as $c\left(\lambda_{0}\right)$. Thus

$$
3 \lambda_{2} c\left(\lambda_{0}\right)=\frac{5}{2 \mu_{1}}\left(\partial_{1}^{2} f\right)-\frac{3}{2} \partial_{1}^{3} f .
$$

We are now ready to calculate the sign of $\varepsilon \int_{\Omega} U^{\prime}(\varepsilon)$ : For small $\varepsilon$ we get, from (4.15),

$$
\operatorname{sign} \varepsilon \int_{\Omega} U^{\prime}(\varepsilon)=\operatorname{sign} \int_{\Omega} U^{\prime \prime}(0) .
$$

By $U(\varepsilon)=\varphi(V(\varepsilon), \Lambda(\varepsilon))$ we get, using (4.15)-(4.17),

$$
\begin{aligned}
\int_{\Omega} U^{\prime \prime}(0) & =\int_{\Omega} \partial_{1}^{2} \varphi v_{1}^{2}+2 \partial_{1} \partial_{2} \varphi v_{1} \lambda_{1}+\partial_{1} \varphi v_{2}+\partial_{2}^{2} \varphi \lambda_{1}^{2}+\partial_{2} \varphi \lambda_{2} \\
& =\partial_{1}^{2} \varphi+\frac{1}{3 \mu_{1}} \partial_{1} \varphi \partial_{1}^{2} f-\frac{4}{3 \mu_{1}} \partial_{1} \varphi \partial_{1}^{2} f-\frac{1}{\mu_{1}} \partial_{1} \varphi \partial_{2} f \lambda_{2}+\partial_{2} \varphi \lambda_{2} \\
& =\partial_{1}^{2} \varphi-\frac{1}{\mu_{1}} \partial_{1} \varphi \partial_{1}^{2} f+\lambda_{2} d\left(\lambda_{0}\right),
\end{aligned}
$$

since $d\left(\lambda_{0}\right)=\left.(d / d \lambda) \varphi(m(\lambda), \lambda)\right|_{\lambda=\lambda_{0}}=-\partial_{1} \varphi \partial_{2} f / \partial_{1} f+\partial_{2} \varphi$. By Corollary 4.4 the stability is determined by the sign of

$$
\begin{aligned}
c\left(\lambda_{0}\right) \int_{\Omega} U^{\prime \prime}(0) & =c\left(\lambda_{0}\right) \frac{\partial_{1} f \partial_{1}^{2} \varphi-\partial_{1} \varphi \partial_{1}^{2} f}{\partial_{1} f}+c\left(\lambda_{0}\right) d\left(\lambda_{0}\right) \lambda_{2} \\
& =\frac{1}{\mu_{1}}\left[c\left(\lambda_{0}\right)\left(\partial_{1} f \partial_{1}^{2} \varphi-\partial_{1} \varphi \partial_{1}^{2} f\right)+\frac{1}{6} d\left(\lambda_{0}\right)\left(5\left(\partial_{1}^{2} f\right)^{2}-3 \partial_{1} f \partial_{1}^{3} f\right)\right] \\
& =\frac{1}{\mu_{1}} J\left(m_{0}, \lambda_{0}\right),
\end{aligned}
$$

using $\partial_{1} f=\mu_{1}$ and (4.18). This proves the theorem.

4.6 EXAMPLE. As the simplest application of the results of this paragraph we will continue the example given in 3.2 for one space dimension, $n=1$ (the result in this case is already known, see [5]),

Here we get

$$
\begin{gathered}
\left(\mu(u, v)\left(u^{\prime}-\chi_{0} u v^{\prime}\right)\right)^{\prime}=0, \quad \nu_{0}\left(v^{\prime \prime}+\alpha u-\beta v\right)=0, \\
u^{\prime}(0)=u^{\prime}(L)=v^{\prime}(L)=0 \quad(L>0) .
\end{gathered}
$$

$$
f(s, \lambda)=\alpha \lambda e^{\chi_{0} s}-\beta s, \quad \varphi(s, \lambda)=\lambda e^{\chi_{0} s} .
$$

From $f(m(\lambda), \lambda)=0$ we get $\varphi(m(\lambda), \lambda)=(\beta / \alpha) m(\lambda)$, and thus

$$
d\left(\lambda_{0}\right)=(\beta / \alpha) m^{\prime}\left(\lambda_{0}\right) \text {. }
$$

Similarly,

$$
c\left(\lambda_{0}\right)=\beta \chi_{0} m^{\prime}\left(\lambda_{0}\right),
$$

where $m^{\prime}\left(\lambda_{0}\right)<0$ (see 3.2). From $f\left(m_{0}, \lambda_{0}\right)=0$ and $\partial_{1} f\left(m_{0}, \lambda_{0}\right)=\mu_{1}$ follows $\alpha \lambda_{0} e^{\chi_{0} m}=\beta m_{0}$ and

$$
\chi_{0} \beta m_{0}=\beta+\mu_{1} .
$$


Thus at $\left(m_{0}, \lambda_{0}\right)$,

$$
\begin{gathered}
\partial_{1} \varphi=\left(\chi_{0} / \alpha\right) \beta m_{0}, \quad \partial_{1}^{2} \varphi\left(\chi_{0}^{2} / \alpha\right) \beta m_{0}, \\
\partial_{1} f=\chi_{0} \beta m_{0}-\beta, \quad \partial_{1}^{2} f=\chi_{0}^{2} \beta m_{0}, \quad \partial_{1}^{3} f=\chi_{0}^{3} \beta m_{0} .
\end{gathered}
$$

So by also using (4.19), (4.20),

$$
J\left(m_{0}, \lambda_{0}\right)=\left(\chi_{0}^{3} \beta^{2} m_{0} m^{\prime}\left(\lambda_{0}\right) / 6 \alpha\right)\left[-6 \beta+5 \chi_{0} \beta m_{0}-3 \mu_{1}\right] .
$$

Since $m^{\prime}\left(\lambda_{0}\right)<0$,

$$
\operatorname{sign} J\left(m_{0}, \lambda_{0}\right)=\operatorname{sign} 6 \beta-5 \chi_{0} \beta m_{0}+3 \mu_{1}=\operatorname{sign} \beta-2 \mu_{1}
$$

because of (4.21). Thus

$$
J\left(m_{0}, \lambda_{0}\right)\left\{\begin{array}{cc}
<0 & \text { if } \beta<2 \mu_{1}, \\
>0 & \text { if } \beta>2 \mu_{1} .
\end{array}\right.
$$

Note that for our problem $\mu_{1}=\pi^{2} / L^{2}$.

RESULT. Solutions of the first bifurcating branch near the bifurcation point are

stable, $\quad$ if $\beta>2 \pi^{2} / L^{2}$,

unstable, if $\beta<2 \pi^{2} / L^{2}$.

More applications of Theorem 4.5 can be found in the examples of $\S 5$.

5. Parametrized global branches and examples. We will consider the case of one space dimension, $\Omega=] 0,1[$, and

$$
\chi(u, v)=\chi_{0} \mu(u, v) u \phi^{\prime}(v)
$$

as described in $\S 1$ (1.2). In our examples, $\phi$ takes the form (1.3)-(1.5). The reaction term will be as simple as possible:

$$
k(u, v)=\nu_{0}(\alpha u-b(v))
$$

with $b(v)=\beta v, \beta>0$, or $b(v)=\beta v /(1+\gamma v), \beta, \gamma>0$. The stationary chemotaxis system then reduces to the scalar problem

$$
v^{\prime \prime}+f(v, \lambda)=0, \quad v^{\prime}(0)=v^{\prime}(1)=1
$$

with

$$
\begin{gathered}
f(v, \lambda)=\alpha \varphi(v, \lambda)-b(v), \\
\varphi(v, \lambda)=\lambda e^{\chi_{0} \phi(v)} .
\end{gathered}
$$

In order to obtain global parametrized branches for (5.1) let us consider a part $\lambda \rightarrow(m(\lambda), \lambda)$ of $M:\{(m, \lambda): f(m, \lambda)=0\}$, where $\partial_{1} f(m(\lambda), \lambda)>0$ (the candidates for bifurcation). For those $\lambda$ the initial value problem

$$
v^{\prime \prime}+f(v, \lambda)=0, \quad v^{\prime}(0)=0, \quad v(0)=a
$$

always has a nonconstant periodic solution $v$ for $a$ in some maximal interval ] $m(\lambda), a^{+}(\lambda)\left[\right.$, where $f(a, \lambda)>0$ (i.e. $v^{\prime \prime}(a)<0$ ). We define the so-called time map $T(a, \lambda)$ then to be half the period of this solution; to be more precise: $T(a, \lambda)$ is defined by

$$
T(a, \lambda)>0, \quad v^{\prime}(T(a, \lambda))=0,\left.\quad v^{\prime}\right|_{|0, T(a, \lambda)|}<0 .
$$

(See Figure 5.1.) 
Because of the symmetry of the problem then $v$ satisfies the boundary condition $v^{\prime}(1)=0$ iff

$$
T(a, \lambda)=1 / j \text { for some integer } j \geqslant 1 \text {. }
$$

Note that (5.4) will also give the solutions of (5.1) with $v^{\prime \prime}(0)>0$, since those correspond to the initial condition $v(0)=\tilde{a}$ (see Figure 5.1). Any solution branch of $T(a, \lambda)=1 / j$ will thus yield two solution branches of nonhomogeneous solutions in $O_{j}$ to (5.1). (For $O_{j}$ see (3.3).)

In [16] we have examined the time map to get parametrized branches to problems as (5.1).

Results to be used in this section are given by Theorems 5.1 and 5.2, where 5.1 recalls some well-known facts about the behaviour of $T$ near $m(\lambda)$ and $a^{+}(\lambda)$ (see also [4]), and 5.2 gives a criterion for $\partial T / \partial a$ to be positive, thus allowing us to use the implicit function theorem to get $C^{1}$-solution curves for (5.4).

5.1 TheOREM. (a) $T(a, \lambda) \rightarrow \pi / \sqrt{\partial_{1} f(m(\lambda), \lambda)}$ as $a \downarrow m(\lambda)$ uniformly on compact $\lambda$-subsets.

(b) If $f\left(a^{+}(\lambda), \lambda\right)=0$ or $f\left(\tilde{a}^{+}(\lambda), \lambda\right)=0$ then $T(a, \lambda) \rightarrow+\infty$ for $a \uparrow a^{+}(\lambda)$.

The assumption of (b) is satisfied in all of our examples. We cannot explain all details about the time map here; for more information the reader is referred to [16]. There we also show that $(\partial / \partial a) T(a, \lambda)$ is positive if e.g. $\partial_{1}^{3} f<0$ and $\partial_{1}^{4} f \geqslant 0$ or $\partial_{1}^{2} f \geqslant 0$, which is a generalization of the result in [18] for the Neumann case. But in fact a better criterion can be given which turns out to hold for all of our examples.

5.2 TheOREM. Let $f(\cdot, \lambda)$ be three times continuous differentiable with

$$
\left(5\left(\partial_{1}^{2} f\right)^{2}-3 \partial_{1} f \partial_{1}^{3} f\right)(s, \lambda)>0 \text { for all }(s, \lambda) \text { with } \partial_{1} f(s, \lambda)>0 \text {, }
$$

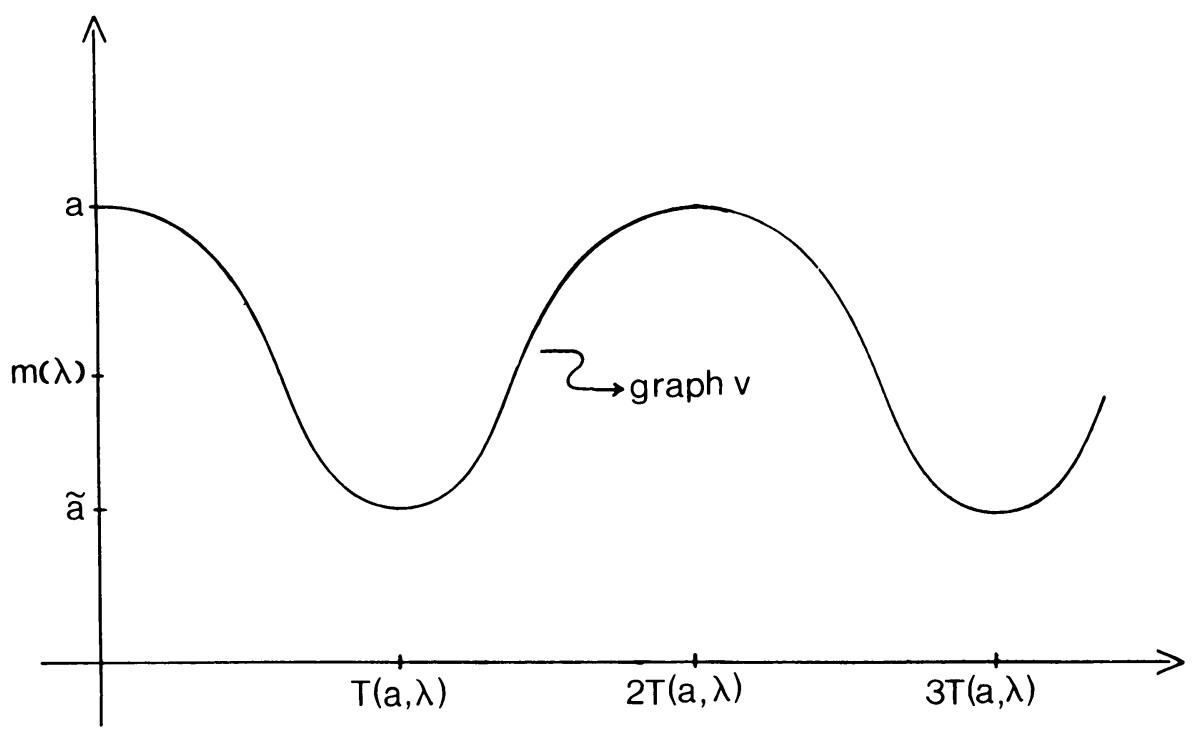

Figure 5.1 
and

$$
\partial_{1}^{2} f(s, \lambda) \cdot(s-m(\lambda))<0 \quad \text { for all }(s, \lambda) \text { with } \partial_{1} f(s, \lambda) \leqslant 0
$$

Then

$$
(\partial / \partial a) T(a, \lambda)>0 \quad \text { for } a \in] m(\lambda), a^{+}(\lambda)[.
$$

The proof of this theorem and others concerning Dirichlet boundary conditions will appear in a forthcoming paper [17]. Note that the term in (5.5) is just the factor of $d\left(\lambda_{0}\right) / 6$ in $J\left(m_{0}, \lambda_{0}\right)$. With the assumptions of Theorems 5.1 and 5.2 satisfied, we can describe the solution set of (5.4) and thus (5.1) in the following way:

$$
\begin{aligned}
& T(a, \lambda)=1 / j \text { has a solution iff } \\
& T(m(\lambda), \lambda)<1 / j \text {, i.e. } \partial_{1} f(m(\lambda), \lambda)>j^{2} \pi^{2}=\mu_{j} .
\end{aligned}
$$

And for each $j$ those solutions form $C^{1}$-curves $(a(\lambda), \lambda)$. The translation to the bifurcation behaviour of (5.1) is given in Figure 5.2.
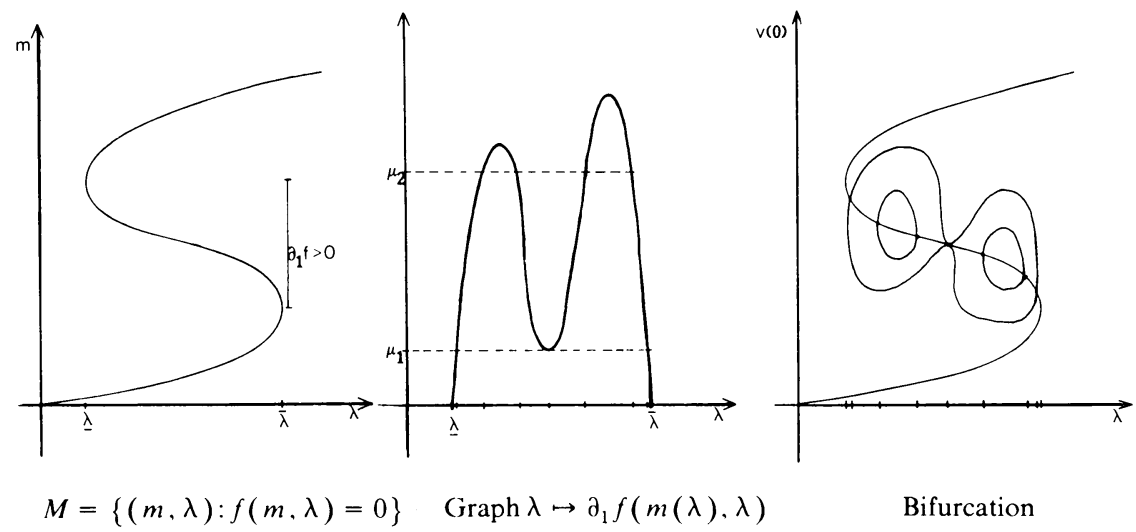

Bifurcation

Figure 5.2

We finish by applying the results to the above mentioned examples. Here we find it more informing to just draw the resulting pictures for each case. The underlying calculations can be easily verified.

5.3 Example. $\phi(v)=v, k(u, v)=\nu_{0}(\alpha u-\beta v), \alpha, \beta>0$.

This example has already been discussed in $\S \S 3$ and 4 . The information to be added is that all branches in fact are $C^{1}$-curves, since the resulting $f(s, \lambda)=\alpha \lambda e^{\chi_{0} s}$ - $\beta s$ satisfies the assumptions of Theorems 5.1 and 5.2.

5.4 Example. $\phi(v)=\ln v, k(u, v)=\nu_{0}(\alpha u-\beta v /(1+\gamma v)) ; \alpha, \beta, \gamma>0$. (The case $\phi(v)=\ln (c+v)$ with $c>0$ can be handled in the same way.) So

$$
f(s, \lambda)=\alpha \lambda s^{\chi_{0}}-\beta s /(1+\gamma s) .
$$

We will here consider the case $1 \leqslant \chi_{0}<2$ for which $\partial_{1}^{3} f<0$, such that (5.5), (5.6) are trivially satisfied.

It may be remarked that our results do not apply to $k(u, v)=\nu_{0}(\alpha u-\beta v)$, since in this case $\partial_{1} f(m, \lambda)$ is a constant along $M$, and no bifurcation occurs. 
$\underline{x_{0}=1}$
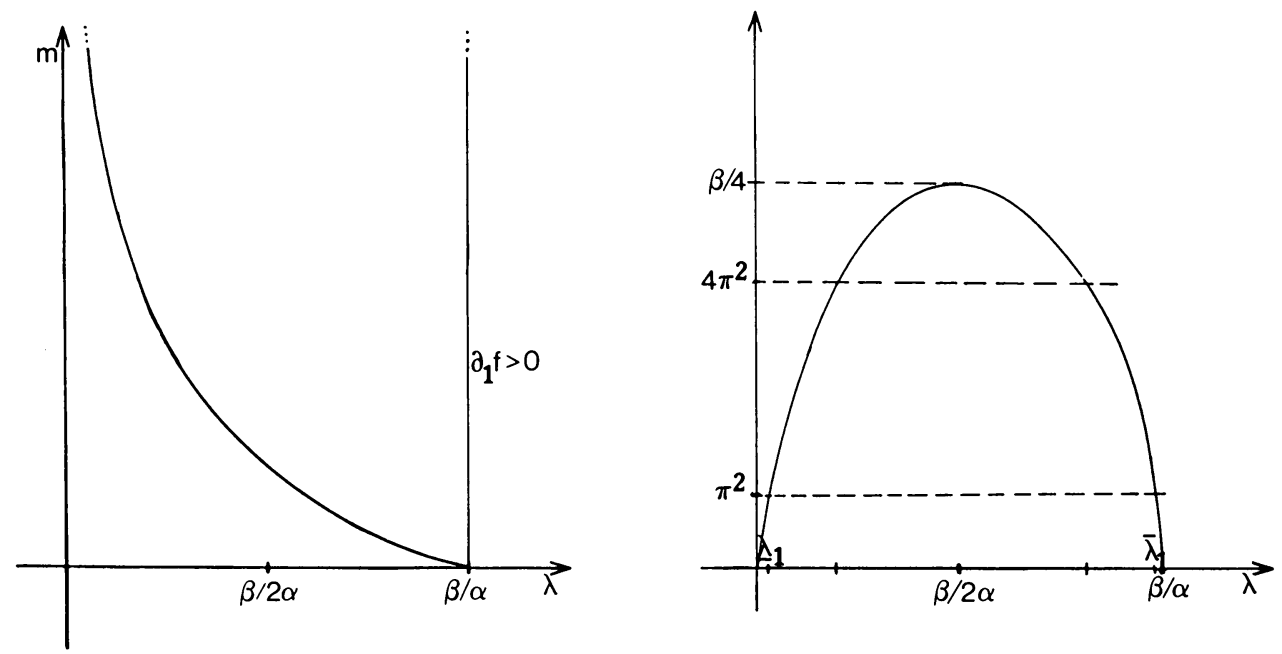

$M=\{(m, \lambda): f(m, \lambda)=0\}$

Graph $\lambda \mapsto \partial_{1} f(m(\lambda), \lambda)$

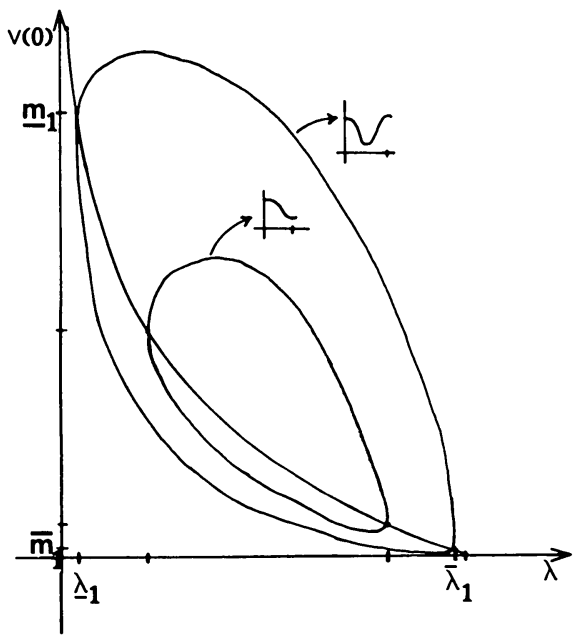

Bifurcation

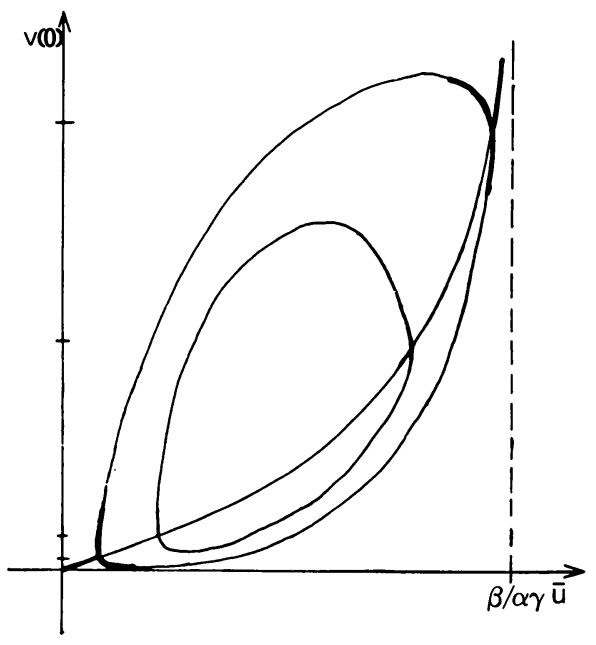

Bifurcation of the system

FIGURE 5.3

The number of bifurcating branches and also the amount of spatial structure in the branches depends on $\beta, \beta>4 \pi^{2}$ is a necessary condition for bifurcation. $\nu_{0} \beta$ is the measure of an amplifying effect on the gradient since it is the degradation rate of the chemical for small $v . \nu_{0}$ is the diffusion constant of the chemical. Thus the amount of spatial structure is increased as $\nu_{0} \beta$ increases for fixed $\nu_{0}$, or as $\nu_{0}$ decreases for fixed $\nu_{0} \beta$.

Regions of stability are marked in the bifurcation diagram for the system. Large and small constant solutions are stable corresponding to the regions where $\partial_{1} f(m(\lambda), \lambda)<\pi^{2}=\mu_{1}$. Bifurcation turns out to be supercritical with respect to $\bar{u}$ 
at both bifurcation points of the first branch such that the branch of monotonic $(u, v)$ starts and ends stable. This is a result of $J\left(\underline{m}_{1}, \underline{\lambda}_{1}\right)$ and $J\left(\bar{m}_{1}, \bar{\lambda}_{1}\right)$ both being negative.

From $f(m(\lambda), \lambda)=0$ we get

$$
(m(\lambda), \lambda)=\frac{\beta}{\alpha} \frac{m(\lambda)}{1+\gamma m(\lambda)},
$$

and thus

$$
d(\lambda)=\frac{d}{d \lambda} \varphi(m(\lambda), \lambda)=\frac{\beta}{\alpha}\left(\frac{1}{1+\gamma m(\lambda)}\right)^{2} m^{\prime}(\lambda)<0 .
$$

In the same way

$$
\partial_{1} f(m(\lambda), \lambda)=\alpha \lambda-\frac{\beta}{(1+\gamma m(\lambda))^{2}}=\frac{\beta}{1+\gamma m(\lambda)}-\frac{\beta}{(1+\gamma m(\lambda))^{2}}
$$

follows. Thus

$$
c(\lambda)=\left(\frac{2 \beta \gamma}{(1+\gamma m(\lambda))^{3}}-\frac{\beta \gamma}{(1+\gamma m(\lambda))^{2}}\right) m^{\prime}(\lambda) .
$$

Furthermore at each point $(m, \lambda)$ in $M$,

$$
\partial_{1}^{2} \varphi \partial_{1} f-\partial_{1} \varphi \partial_{1}^{2} f=-\lambda \frac{2 \beta \gamma}{(1+\gamma m)^{3}}=-\frac{2 \beta^{2} \gamma}{\alpha} \frac{1}{(1+\gamma m)^{4}}<0 .
$$

For the bifurcation point $\left(\underline{m}_{1}, \underline{\lambda}_{1}\right)$ we have $c\left(\underline{\lambda}_{1}\right)>0$ (see Figure 5.3), $d\left(\underline{\lambda}_{1}\right)<0$, the factor of $c\left(\underline{\lambda}_{1}\right)$ in $J\left(\underline{m}_{1}, \underline{\lambda}_{1}\right)$ is negative (5.9), the one of $d\left(\underline{\lambda}_{1}\right)$ is positive since (5.5) is satisfied. So $J\left(\underline{m}_{1}, \underline{\lambda}_{1}\right)$ is negative. For $J\left(\bar{m}_{1}, \bar{\lambda}_{1}\right)$ the terms have oppositive sign, so calculations become a bit more involved. With $A:=1 /\left(1+\gamma \bar{m}_{1}\right)<1$ we get from (5.7), (5.8)

(5.9) gives

$$
d\left(\bar{\lambda}_{1}\right)=-A^{2} m^{\prime}\left(\bar{\lambda}_{1}\right), \quad c\left(\bar{\lambda}_{1}\right)=\beta \gamma A^{2}(2 A-1) m^{\prime}\left(\bar{\lambda}_{1}\right) .
$$

$$
\left(\partial_{1}^{2} \varphi \partial_{1} f-\partial_{1} \varphi \partial_{1}^{2} f\right)\left(\bar{m}_{1}, \bar{\lambda}_{1}\right)=-\left(2 \beta^{2} \gamma / \alpha\right) A^{4} .
$$

Using $f\left(\bar{m}_{1}, \bar{\lambda}_{1}\right)=0$ and $\partial_{1} f\left(\bar{m}_{1}, \bar{\lambda}_{1}\right)=\pi^{2}$ we can calculate

$$
\frac{1}{6}\left(5\left(\partial_{1}^{2} f\right)^{2}-3 \partial_{1} f \partial_{1}^{3} f\right)\left(\bar{m}_{1}, \bar{\lambda}_{1}\right)=\frac{10}{3} \beta^{2} \gamma^{2} A^{6}+3 \pi^{2} \beta \gamma^{2} A^{4} .
$$

Thus

$$
J\left(\bar{m}_{1}, \bar{\lambda}_{1}\right)=-m^{\prime}\left(\bar{\lambda}_{1}\right)\left(\beta^{2} A^{6} \gamma^{2} / 3 \alpha\right)\left[6 \beta(2 A-1)-10 \beta A^{2}-9 \pi^{2}\right] .
$$

The sign of $J\left(\bar{m}_{1}, \bar{\lambda}_{1}\right)$ is given by the term in square brackets. Since $\pi^{2}=$ $\partial_{1} f\left(\bar{m}_{1}, \bar{\lambda}_{1}\right)=\beta\left(A-A^{2}\right)$ this one simplifies to $2 \beta A-6 \beta+\pi^{2}<\pi^{2}-4 \beta$ since $A<1$. The last term is certainly negative since $\beta \geqslant 4 \pi^{2}$ is a necessary condition for bifurcation.

$1<\chi_{0}<2$.

$M$ and the graph $\lambda \mapsto \partial_{1} f(m(\lambda), \lambda)$ are drawn in Figure 5.4.

Therefore we get two different situations:

$\beta\left(\chi_{0}-1\right)<\pi^{2}$. See Figure 5.5.

$\underline{\beta\left(\chi_{0}-1\right) \geqslant \pi^{2}}$. See Figure 5.6. 

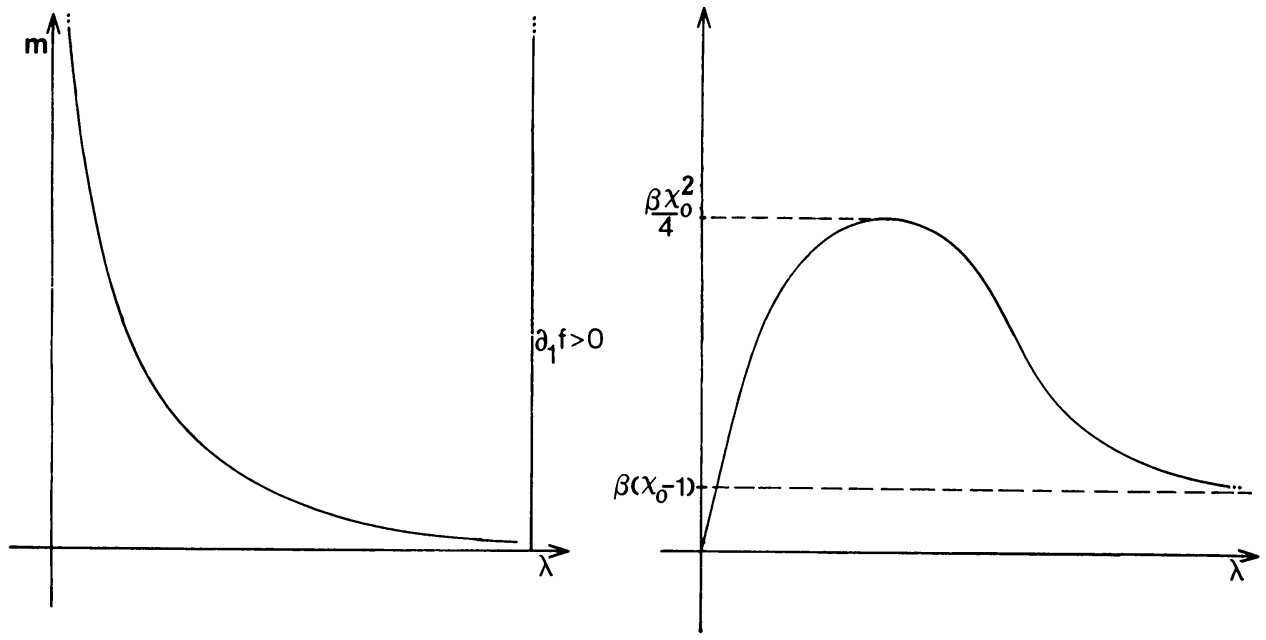

FIGURE 5.4
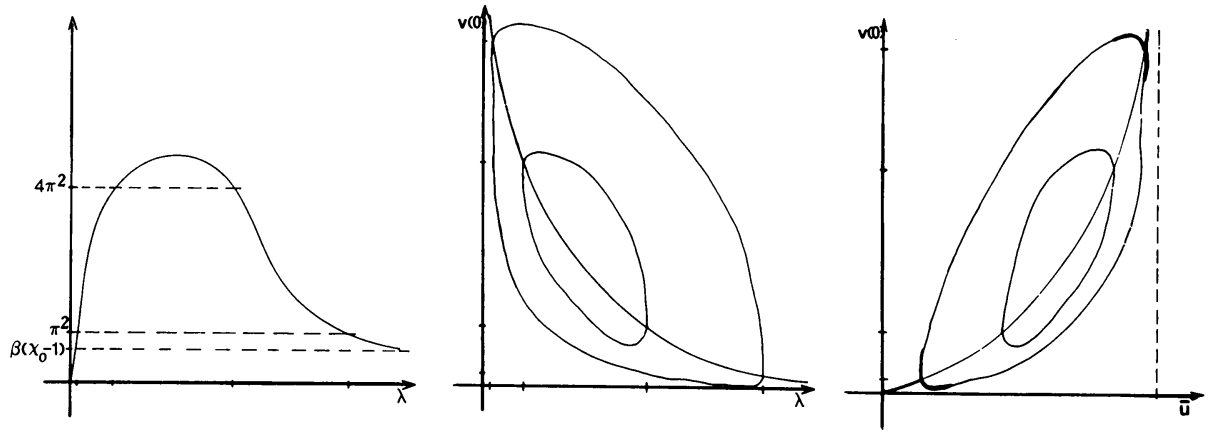

FigURE 5.5
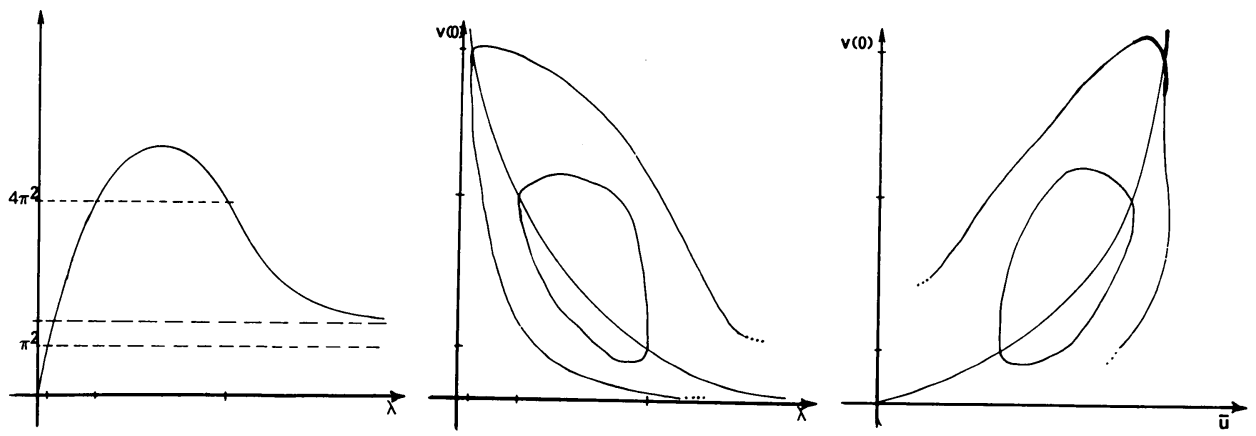

FigURE 5.6 
Here the number of bifurcating branches is determined by the maximum $\beta \chi_{0}^{2} / 4$, the interpretation is the same as in the case $\chi_{0}=1$. Regions of stability are marked; calculations that $J\left(\underline{m}_{1}, \underline{\lambda}_{1}\right)$ and $J\left(\bar{m}_{1}, \bar{\lambda}_{1}\right)$ have the right sign can be done similarly to the case $\chi_{0}=1$, but become rather involved and are therefore omitted.

5.5 Example. $\phi(v)=v /(1+c v), c>0, k(u, v)=\nu_{0}(\alpha u-\beta v)$. Here

$$
f(s, \lambda)=\alpha \lambda \exp \left(\chi_{0} s /(1+c s)\right)-\beta s .
$$

Only, for $\chi_{0}>4 c$, there is a part of the zero set $M$ where $\partial_{1} f>0$. Calculations to show (5.5) and (5.6) become more lengthy but straightforward. The bifurcation result is given in Figure 5.7.
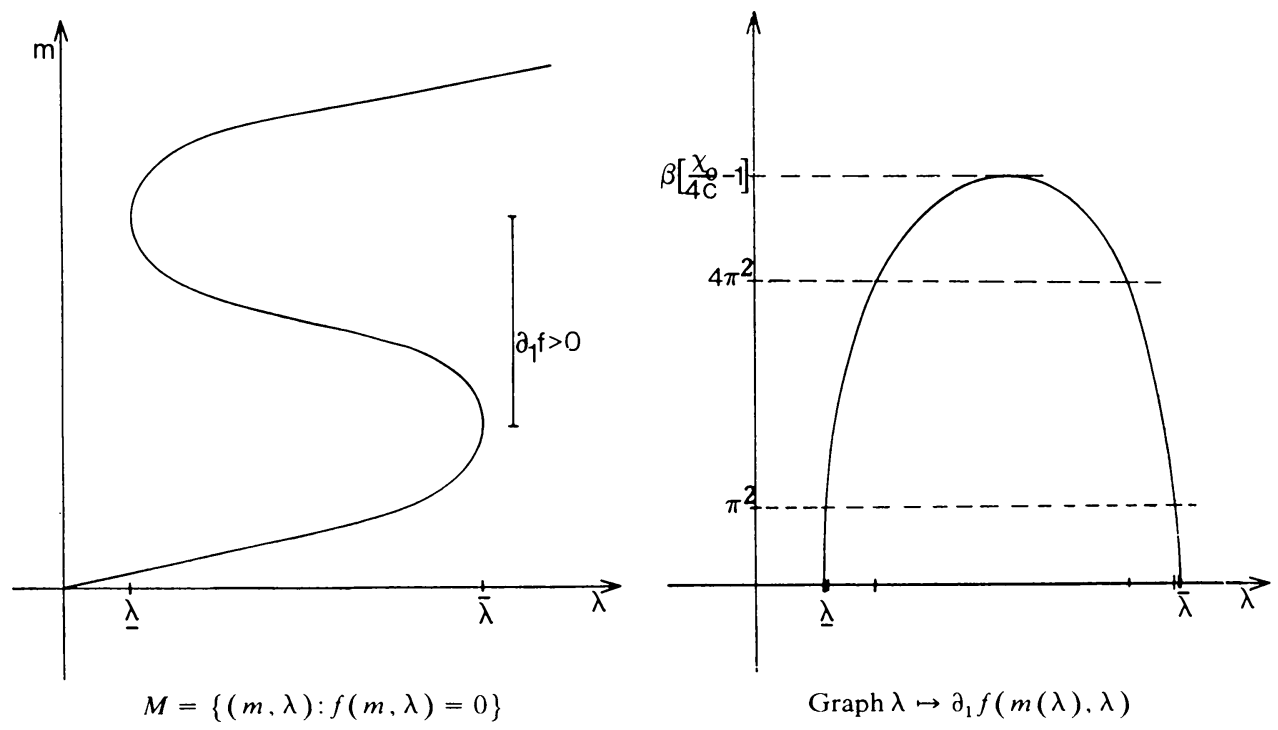

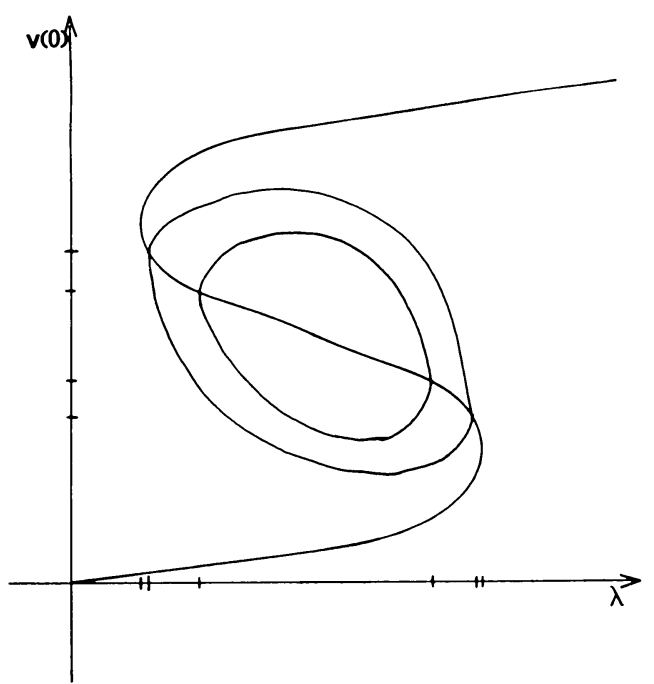

Bifurcation

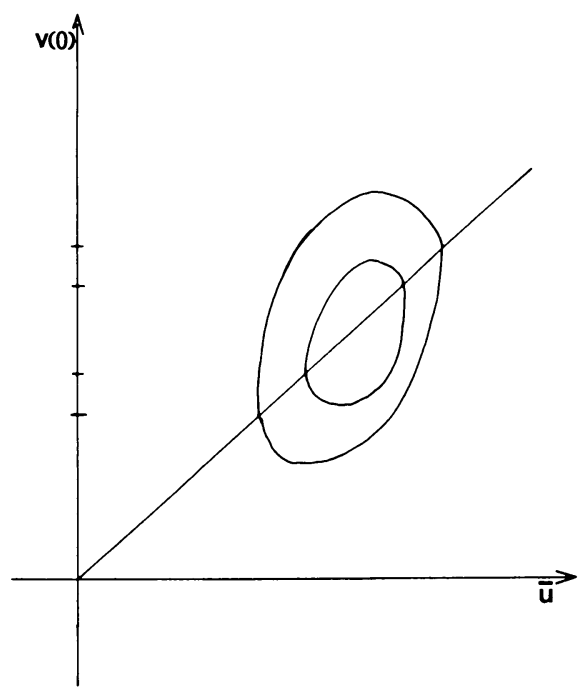

Bifurcation of the system

FIGURE 5.7 
$\beta\left(\chi_{0} / 4 c-1\right)>\pi^{2}$ becomes the condition for bifurcation. There is at least a subrange of the specified parameter region within which there are stable bifurcating branches. But calculations become rather complicated. In cases like this it seems more appropriate to test the sign of $J(m, \lambda)$ numerically.

As a last remark we will give the first step towards the investigation of stability along the global first solution branch. In $\$ 2$ we have shown that

$$
G(u, v)=\left(\begin{array}{c}
\left(\mu(u, v) u^{\prime}-\chi(u, v) v^{\prime}\right)^{\prime} \\
\nu_{0} v^{\prime \prime}+k(u, v)
\end{array}\right)=0
$$

is equivalent to

$$
H(v, \lambda)=v^{\prime \prime}+f(v, \lambda)=0, \quad u=\varphi(v, \lambda),
$$

$G: B \times B \rightarrow Y \times Y, H: B \times \mathbf{R}^{+} \rightarrow Y$. If now $f$ satisfies the conditions for $(\partial / \partial a) T(a, \lambda)>0$ it can be shown (see $[19,20])$ that the linearization $D_{v} H(v, \lambda)$ is always nonsingular for $(v, \lambda)$ on a bifurcating branch, i.e. eigenvalues of $D_{v} H$ do not cross the imaginary axis along a global bifurcating branch $\lambda \rightarrow(V(\lambda), \lambda)$ of (5.11)

Let $\lambda \rightarrow((U(\lambda), \lambda), V(\lambda))$ be the corresponding branch of $(5.10), U(\lambda)=$ $\varphi(V(\lambda), \lambda)$. Then by Theorem 2.2,

$$
D G((U(\lambda), \lambda), V(\lambda))(u, v)=0 \text { iff } u=\partial_{1} \varphi(V(\lambda), \lambda) v+\eta \partial_{2} \varphi(V(\lambda), \lambda)
$$

for some $\eta \in \mathbf{R}$, and $D_{v} H(V(\lambda), \lambda) v+\eta \partial_{2} f(V(\lambda), \lambda)=0$.

Since $D_{v} H(V(\lambda), \lambda)$ is nonsingular we get that $v=\eta V^{\prime}(\lambda)$ from $(d / d \lambda) H(V(\lambda), \lambda)=0$. So $u=\eta\left(\partial_{1} \varphi(V(\lambda), \lambda) V^{\prime}(\lambda)+\partial_{2} \varphi(V(\lambda), \lambda)\right)=\eta U^{\prime}(\lambda)$. Thus the nullspace $N(D G(U(\lambda), \lambda))$ is always one dimensional and is spanned by $\left(U^{\prime}(\lambda), V^{\prime}(\lambda)\right)$.

Now there are two possibilities for a change of stability along a branch $(U(\lambda), V(\lambda))$. It might be due to a pair of complex conjugate eigenvalues crossing the imaginary axis, in which case (in general) there would be Hopf bifurcation. This can be excluded in special examples (e.g. Example 5.3). The other possibility would be that for some $\lambda$ the linearization $D G\left(U(\lambda), V(\lambda)\right.$ ) has a nullspace in $X_{0} \times X$. According to above calculations this is only possible if $(d / d \lambda) \int_{\Omega} U(\lambda)=0$, i.e. at a turning point of the branch with respect to the parameter $\bar{u}=\int_{\Omega} u$.

\section{REFERENCES}

1. J. C. Alexander and Stuart S. Antman, Global and local behaviour of bifurcating multidimensional continua of solutions for multiparameter nonlinear eigenvalue problems, Arch. Rational Mech. Anal. 76 (1981), 339-354.

2. W. Alt, Biased random walk models for chemotaxis and related diffusion approximations, J. Math. Biol. 9 (1980), 147-177.

3. , Orientation of cells migrating in a chemotactic gradient, Biological Growth and Spread, Proceedings, Heidelberg 1979, Lecture Notes in Biomathematics, vol. 38, Springer-Verlag, 1980.

4. N. Chafee and E. F. Infante, A bifurcation problem for a nonlinear partial differential equation of parabolic type, Applicable Anal. 4 (1974), 17-37.

5. S. Childress and J. K. Percus, Nonlinear aspects of chemotaxis, Math. Biosci. 56 (1981), 217-237.

6. M. Crandall and P. Rabinowitz, Bifurcation from simple eigenvalues, J. Funct. Anal. 8 (1973), $161-180$.

7. , Bifurcation, perturbation of simple eigenvalues and linearized stability, Arch. Rational Mech. Anal. 52 (1973), 161-181. 
8. G. L. Hazelbauer (ed.), Taxis and behaviour, elementary sensory systems in biology, Receptors and Recognition, Series B, vol. 5, Chapman and Hall, London, 1978.

9. T. Kato, Perturbation theory for linear operators, Springer-Verlag, 1980.

10. E. F. Keller and L. A. Segel, Initiation of slime mold aggragation viewed as an instability, J. Theoret. Biol. 26 (1970), 399-415.

11. H. Kielhöfer, On the Lyapunov-stability of stationary solutions of semilinear parabolic differential equations, J. Differential Equations 22 (1976), 193-208.

12. I. R. Lapidus and M. Levandowsky, Modeling chemosensory responses of swimming eukarvotes, Biological Growth and Spread, Proceedings, Heidelberg 1979, Lecture Notes in Biomathematics, vol. 38 , Springer-Verlag, 1980.

13. V. Nanjundiah, Chemotaxis, signal relaying and aggregation morphology, J. Theoret. Biol. 42 (1973), 63-105.

14. M. Protter and H. Weinberger, Maximum principles in partial differential equations, Prentice-Hall, Englewood Cliffs, N. J., 1967.

15. P. Rabinowitz, Some global results for nonlinear eigenvalue problems, J. Funct. Anal. 7 (1971), 487-513.

16. R. Schaaf, Global behaviour of solution branches for some Neumann problems depending on one or several parameters, J. Reine Angew. Math. 346 (1984), 1-31.

17. Global solution branches via the time maps, SFB 123, preprint (1985).

18. J. Smoller and A. Wasserman, Global bifurcation of steady state solutions, J. Differential Equations 39 (1981), 269-290.

19. J. Smoller, A. Tromba and A. Wasserman, Nondegenerate solutions of boundary value problems, J. Nonlinear Analysis 4 (1980), 207-216.

20. J. Smoller, Shock waves and reaction-diffusion equations, Springer, 1983.

21. R. P. Sperb, On a mathematical model describing the aggregation of amoebae, Bull. Math. Biol. 41 (1979), 555-571.

Universität Heidelberg, Sonderforschungsbereich 123, Im Neuenheimer Feld 294, D - 6900 Heidelberg, Federal Republic of Germany 